\title{
A Fully Automatic Nerve Segmentation and Morphometric Parameter Quantification System for Early Diagnosis of Diabetic Neuropathy in Corneal Images
}

\author{
Shumoos Al-Fahdawi ${ }^{1}$, Rami Qahwaji ${ }^{1}$, Alaa S. Al-Waisy ${ }^{1}$, Stanley Ipson ${ }^{1}$, Rayaz A. Malik ${ }^{2,3}$, Arun \\ Brahma $^{4}$, Xin Chen ${ }^{5}$ \\ ${ }^{1}$ School of Electrical Engineering and Computer Science, University of Bradford, Bradford, UK. \\ ${ }^{2}$ Division of Medicine, Weill Cornell Medical College in Qatar, Doha, Qatar. \\ ${ }^{3}$ Centre for Endocrinology and Diabetes, Institute of Human Development, University of Manchester and the Manchester Royal Infirmary, Central Manchester \\ Hospital Foundation Trust, Manchester, U.K. \\ ${ }^{4}$ Manchester Royal Eye Hospital, Central Manchester University Hospitals NHS Foundation Trust, Manchester Academic Health Science Centre, Manchester, \\ M13 $9 W L, U K$. \\ ${ }^{5}$ Centre for Imaging Sciences, Institute of Population Health, University of Manchester, Manchester, U.K.
}

\section{ABSTRACT}

Diabetic Peripheral Neuropathy (DPN) is one of the most common types of diabetes that can affect the cornea. An accurate analysis of the nerve structures can assist the early diagnosis of this disease. This paper proposes a robust, fast and fully automatic nerve segmentation and morphometric parameter quantification system for corneal confocal microscope images. The segmentation part consists of three main steps. Firstly, a preprocessing step is applied to enhance the visibility of the nerves and remove noise using anisotropic diffusion filtering, specifically a Coherence filter followed by Gaussian filtering. Secondly, morphological operations are applied to remove unwanted objects in the input image such as epithelial cells and small nerve segments. Finally, an edge detection step is applied to detect all the nerves in the input image. In this step, an efficient algorithm for connecting discontinuous nerves is proposed. In the morphometric parameters quantification part, a number of features are extracted, including thickness, tortuosity and length of nerve, which may be used for the early diagnosis of diabetic polyneuropathy and when planning Laser-Assisted in Situ Keratomileusis (LASIK) or Photorefractive keratectomy (PRK). The performance of the proposed segmentation system is evaluated against manually traced ground-truth images based on a database consisting of 498 corneal sub-basal nerve images (238 are normal and 260 are abnormal). In addition, the robustness and efficiency of the proposed system in extracting morphometric features with clinical utility was evaluated in 919 images taken from healthy subjects and diabetic patients with and without neuropathy. We demonstrate rapid (13seconds/image), robust and effective automated corneal nerve quantification. The proposed system will be deployed as a useful clinical tool to support the expertise of ophthalmologists and save the clinician time in a busy clinical setting.

Keywords: Diabetes, Diabetic Peripheral Neuropathy, Corneal Confocal Microscopy, Corneal Subbasal Epithelium, Automatic Nerve Segmentation, Anisotropic Diffusion Filtering.

\section{Introduction}

The cornea consists of five layers: the Epithelium layer, Bowman's layer, the Stroma, Decement's membrane layer and the Endothelium layer [1] (Fig.1). It contains sensory and autonomic nerves located at the interface between the Bowman's layer and the basal epithelium. Corneal Confocal Microscopy $(\mathrm{CCM})$ is a rapid non-invasive in vivo clinical technique for capturing images of the different corneal layers [2]. Morphological alterations in the epithelium, stroma and endothelium provide insights into a variety of corneal diseases $[3,4]$ and assessment of the effects of wearing contact lenses [1], LASIK or PRK [5], fungal keratitis [6], corneal transplantation [7] or conditions such as keratoconus [8,9]. CCM has also been used in the assessment of peripheral neuropathies $[10,11,12,13,14,15,16,17,18,19]$. The development of automated imaging algorithms for the processing of CCM images $[20,21,22,23]$ is a necessary accompaniment to such work. Diabetes is the main cause of neuropathy complications and in the present work patients with a known history of cancer, chemotherapy, alcoholism, celiac disease or a deficiency of vitamin B12 or folate, abnormality in ANA or immunoglobulins were excluded to remove other causes of peripheral neuropathy from the input data. Diabetes can result in nerve disorders and nerve damage that affect various parts of the human body, such as the digestive tract and the cardiovascular system $[15,17]$. Quantifying corneal nerve morphology has been shown to have promise as an imaging biomarker for early diagnosis of subclinical diabetic neuropathy $[11,12,17,18]$ and to have value in predicting those who develop clinical neuropathy [16,24] and response to therapy [25]. Presently, most analysis methods of the corneal nerves are based on wearisome and very time consuming manual tracing programs $[5,6,217,26]$. As a result, the information obtained on the clinical parameters quantification is subjective and can have limited reproducibility [27]. A fully automatic and real-time system for tracing sub-basal nerves and extracting clinically meaningful parameters is required. Such a system would reliably and efficiently assess nerve pathology in diabetic patients and provide an objective means for diagnostic and staging purposes $[28,29,30]$. However, in order to build an efficient and robust system for segmenting the sub-basal nerves in corneal images, a number of issues need to be taken into account, including the visual contrast of the nerves, the discontinuities in some nerve images, and the inconsistent intensities of corneal sub-basal images, which can all play a significant role in decreasing segmentation performance. 
In this paper, a robust, fully automatic segmentation and morphometric parameter quantification system for CCM images of human corneal sub-basal nerves is proposed. The segmentation part consists of three main steps. Firstly, a preprocessing step to enhance the visibility of the nerves and reduce noise by applying anisotropic diffusion filtering (Coherence filter) and a Gaussian filter. Secondly, unwanted features such as epithelial cells and other small structures, which are not nerves, are removed from the input image by applying a number of morphological operations. Finally, an edge detection process is applied to detect all the nerves in the input image. In the quantification of morphometric parameters a number of useful clinical features are extracted, including tortuosity, length and thickness of the nerve to aid in the early diagnosis of DPN.

The main contribution of this work is developing an objective and fully automatic system that can be used for tracing the subbasal nerves in corneal images and extracting meaningful clinical features for early diagnosis of diabetic neuropathy. Moreover, an efficient algorithm is proposed for connecting discontinuous nerves without any manual intervention, which can play a significant role in calculating helpful and meaningful clinical features, such as the nerve tortuosity and the nerve length that mainly depend on the whole structure of the nerve. Finally, an efficient and accurate nerve thickness algorithm, without any manual intervention, is also proposed.

This paper is organized as follows: Related work is discussed in Section 2. Section 3 includes descriptions of the materials used, and the proposed corneal sub-basal nerve segmentation and quantification system. The experimental results are presented in Section 4. Finally, conclusions and future research directions are stated in the last section.

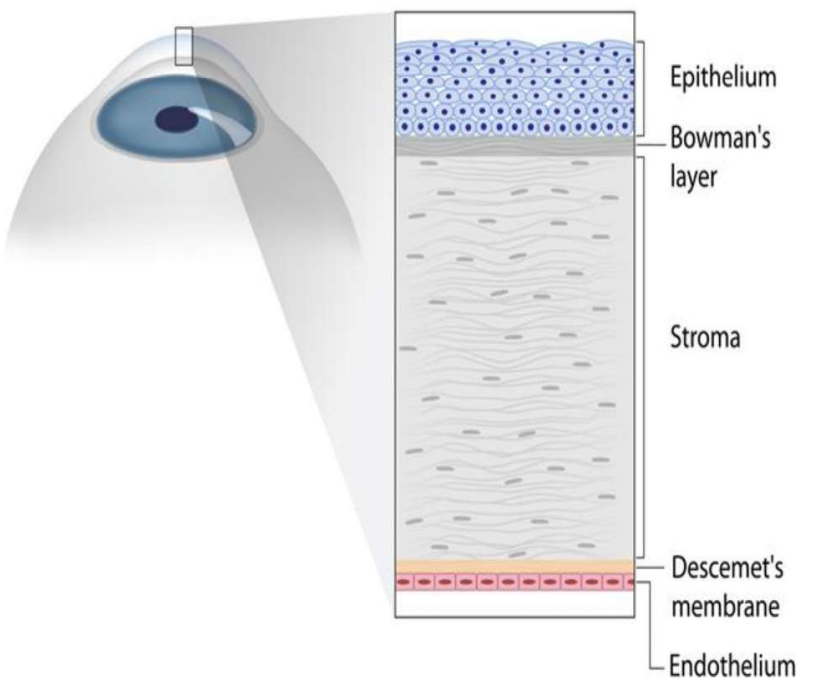

Figure 1. The five distinct layers of the cornea [31].

\section{Related Work}

Over the last few years, increased demand for a fully automatic segmentation system for corneal nerves has led to interesting developments in this field. However, most of the systems proposed are incapable of detecting the corneal nerves without the aid of manual support such as the provision of seed points. The work proposed in [28] is a phase symmetry-based system for the segmentation of corneal nerves of images acquired by corneal confocal microscopy. Firstly, the contrast of the nerves is increased and the noise is reduced, using contrast equalization, a phase symmetry-based approach and histogram processing. This is followed by a region growing, nerve reconstruction, technique to join the disconnected nerves. Small, isolated segments are discarded. Finally, two morphometric parameters are measured, which are nerve tortuosity and nerve length. The method was tested on a small dataset consisting of 15 images and achieved $87 \% \pm 8 \%$ correctly segmented nerves. The authors in [26] used the same algorithm as in [28] but with a different dataset to extract tortuosity, density, length, width and branching. These were used to identify the presence and severity of diabetic peripheral neuropathy. However, the authors found that the proposed nerve segmentation was limited in terms of accurately extracting the clinical features.

Ruggeri and et al. [32] proposed a corneal nerve tracing and recognition system. The system starts by normalizing the contrast and luminosity of a corneal image and then applies an averaging filter to reduce its noise. A tracking procedure is then implemented starting from a set of automatically defined seed points. In the final stage, fuzzy c-mean clustering is applied to classify the pixels as "nerve" or "non-nerve" pixels. The system performance was tested on 12 images and the execution time was 4-5 minutes per image, but there are no further details about how they measured execution time. The results showed that the performance of the system could be affected by the presence of cells in images. Epithelial cells were incorrectly identified as segments of nerves. Poletti and Ruggeri [33] presented an algorithm for corneal nerve recognition based on an automatically identified set of seed points lying all over the image. The nerves are traced by connecting the seed points using their minimum cost paths. This system was tested using a dataset consisting of 30 epithelium corneal images. The algorithm achieved an average sensitivity of about 0.85 , a false detection rate of 0.05 and an execution time of 25 seconds per image, but again, further details about how the execution time was measured are not available.

Ferreira and et al. [34] developed an automatic segmentation and morphometric analysis system for sub-basal corneal nerve images obtained by in vivo corneal confocal microscopy. The system starts by enhancing the image contrast using an adaptive histogram equalization method. To identify the nerve structures, a phase symmetry-based algorithm using a wavelet transform filter was used. Then, a nerve reconstruction process was implemented using a manually selected set of seed points, followed by a sequence of morphological operations to discard small segments. The system failed to recognize $5.3 \%$ of the nerves correctly. Scarpa and et al. [35] presented an algorithm for detecting corneal nerves in CCM images. Firstly, the algorithm starts by enhancing the luminosity and contrast of the corneal images by employing an equalization technique. This is followed by an automatic procedure to identify a set of seed points all over the input image to be used as starting points to detect each nerve in the image. The algorithm was tested on a dataset consisting of 90 images of control subjects and patients. It correctly recognized $80.4 \%$ and $83.8 \%$ of nerve length, compared with the manually traced nerve length, in control subjects and patients, respectively. Scarpa and et al. [36] presented an automatic algorithm to calculate and classify the tortuosity of corneal nerves using a dataset containing 30 corneal sub-basal nerve images. The proposed algorithm depends on the tracing and recognition system for corneal nerves in [35]. The nerve tortuosity was calculated using the proposed algorithm based on the number of twists in the curvature sign and on the amplitude. The results obtained were compared against the manual evaluation performed by an expert. Only 2 of the 30 images were misclassified using the proposed algorithm. Dabbah et al. [37] developed two techniques for nerve fiber detection. The first technique is based on a linear operator, which was devised originally for asbestos fibers. This technique exploits the line-like structure of the nerve. The second technique is based on Gabor wavelet filtering to detect nerve fibers in the corneal image, with 
a thresholding operation following both methods to obtain binary images which are thinned to provide skeleton images with a tolerance of about $\pm 3.1 \mu \mathrm{m}$ in nerve location. The two proposed techniques were tested on only $12 \mathrm{CCM}$ images, which have limited ability to give a clear indication of the performance of the proposed techniques.

An automatic analysis and classification system for detecting nerves in confocal microscopy corneal images based on a multiscale dual model detection algorithm is presented in [29]. Feature vectors are generated from this dual-model detection to be used in the classification stage, which is based on the Random Forest (RF) and Neural Networks (NN) to classify the pixels as nerve or non-nerve pixels. The performance of the proposed system was evaluated using database consisting of $521 \mathrm{CCM}$ images, which is a subset of the second database used here. The proposed system achieved highest sensitivity and specificity at the Equal Error Rate (EER) of $15.44 \%$. The work presented in [38] used an automatic system to trace sub-basal plexus nerves in images acquired by CCM. First a top-hat Morphological operation was used to enhance the image's contrast. Next, a Log-Gabor filter was applied to enhance the corneal nerve structure. This was followed by hysteresis thresholding to obtain candidate nerve segments that were input to a Support Vector Machine (SVM) to distinguish between nerve and non-nerve segments. The system performance was tested on 246 images and achieved an average sensitivity of $0.88 \pm 0.06$. A supervised learning algorithm to classify CCM images based on manually traced tortuosity of the nerves was introduced in [39]. The method was applied to 100 corneal nerve images and tortuosity was classified into four classes (normal, mild, moderate and severe) by three ophthalmologists. Curvature and number of inflection points were used in the feature vector for the proposed supervisedlearning system. In this system, a training phase is needed and the results obtained do not meet the desired level.

Through this review of the research on corneal nerve tracing and clinical features extraction it is clear that there is a significant need for improved methodology to quantify corneal nerve morphology. Firstly, most of the existing methods are based on defining a set of seed points all over the image, whether manually or automatically. Secondly, relatively small datasets of CCM images have been employed in the evaluation of most of these methods.
Moreover, the processing time for tracing all the nerves in a single image can take more than one minute in some cases. To overcome these deficiencies, an accurate, fast and fully automatic corneal nerve segmentation system is proposed here.

\section{Materials and Methods}

The proposed automatic nerve evaluation system is divided into two main stages: the nerve segmentation stage and the morphometric parameters quantification stage. The segmentation stage consists of three main steps: a preprocessing step to enhance the images; a morphological operations step to remove unwanted objects and an edge detection step to detect the nerves. In the morphometric parameter quantification stage, the clinically useful nerve features, thickness, tortuosity, length and density, are extracted and presented in a quantitative format. A block diagram of the proposed system is shown in Fig. 2.

\subsection{Image Acquisition}

In this paper, two databases were used to assess the efficiency of the proposed system. The first consists of a total of 498 images from 20 subjects [26] and the number of images per subject varies between 12 and 38. 12 subjects had diabetes and 8 had no diabetes, with mean ages of $58 \pm 10$ years and $54 \pm 7$ years, respectively. Diabetic patients were classified into: 4 with No neuropathy (mean age $53 \pm 11$ years), 5 with Mild Neuropathy (mean age $58 \pm 9$ years) and 3 with Moderate neuropathy (mean age $60 \pm 9$ years). Images (Fig.3) were acquired using a Heidelberg Retinal Tomograph equipped with a Cornea Rostock Module (HRT-CRM: Heidelberg Engineering, Heidelberg, Germany). The images were saved in JPEG compressed format with a size of $(384 \times 384)$ pixels covering a $400 \mu \mathrm{m} \times 400 \mu \mathrm{m}$ frame size at an optical magnification of $63 \mathrm{X}$. The second database [14] consists of a total of 919 images where 445 images are from 84 control subjects and the remaining 350 images are from 63 diabetic patients without neuropathy, and 124 images are from 25 patients with neuropathy. The CCM images were captured using a Heidelberg Retina Tomograph equipped with Rostock Cornea Module (HRT-III). The images have a size of $(384 \times 384)$ pixels with a pixel size of $1.0417 \mu \mathrm{m}$ and 8-bit grey levels and were saved in BMP format.

The Nerve Segmentation Stage

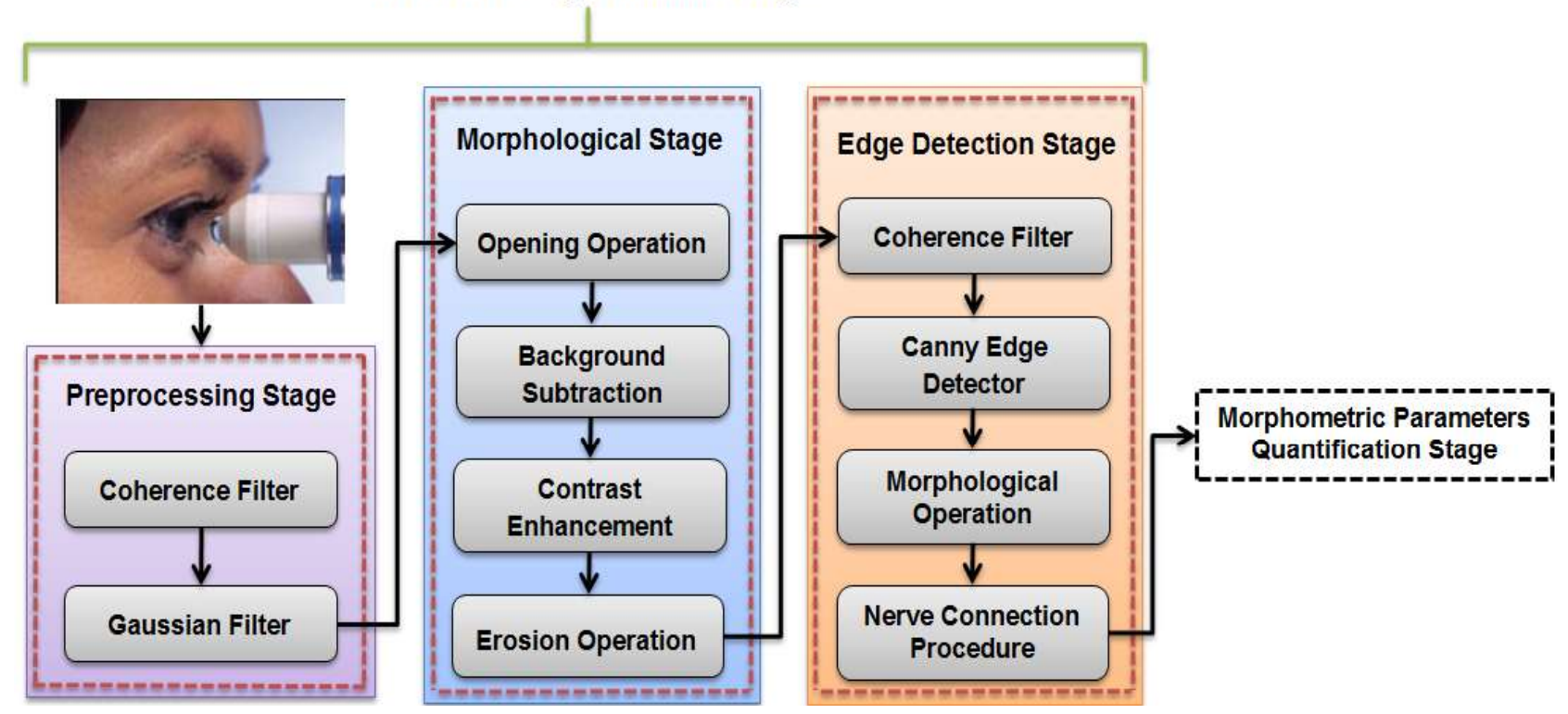

Figure 2. Overview of the automatic proposed corneal nerve segmentation and quantification system. 

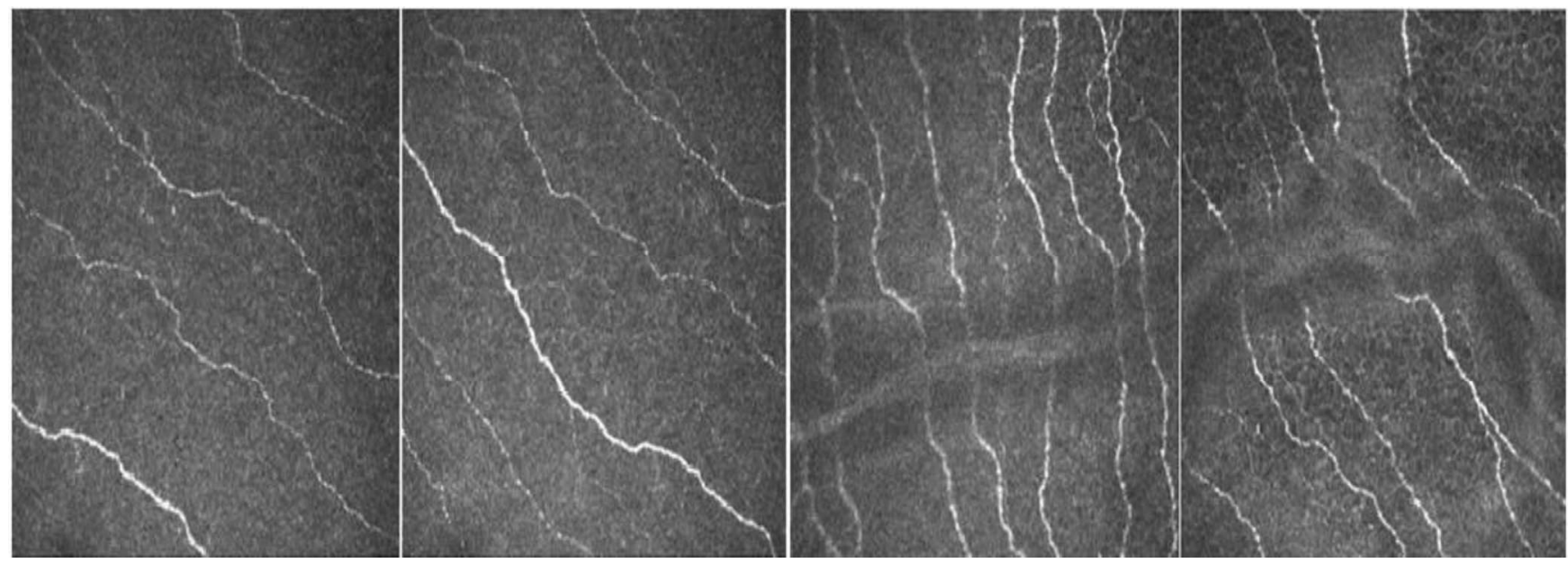

Figure 3. Images from the dataset used:The first two images from the left side are from healthy control subjects, while the last two images are from diabetic patients.

\subsection{Preprocessing Stage}

The preprocessing stage aims to address a number of issues related to enhancing and improving the quality of the corneal images. Movements of the eye during the image acquisition process can cause a motion blurring effect and those CCM images of adjacent layers to be displaced laterally with respect to each other. In addition, the spherical shape of the cornea layer leads to unequal distribution of lighting in the different areas of the cornea during the acquisition process. The image acquisition process can also lead to the emergence of some observed artefacts.

In order to address all these problems, coherence filtering (anisotropic diffusion filtering) and Gaussian filtering have been used to enhance edges in the corneal image and reduce noise while preserving the nerve structure. In this paper, a tensor form was used to adjust the diffusion to the underlying corneal image structure and reduce the noise along the edges of the nerves, rather than using a scalar diffusion constant. In general, the tensor can be built in many ways. In this work, either CoherenceEnhancing Diffusion (CED) for enhancing line-like textures in the image or Edge-Enhancing Diffusion (EED) to reduce the noise while enhancing edges is used [40,41]. In this paper, a hybrid diffusion filter with a Continuous Switch (HDCS) has been used, combining the CED and EED algorithms [40]. The HDCS is an important approach for nerve enhancement, because the corneal image contains tubular and planar image structures. Therefore, if the structure of the image is tubular, the HDCS turns into CED and if it is planar, the HDCS turns into EED. In addition, the standard discretization scheme, nonnegative discretization scheme and the optimized discretization scheme have been used as diffusion schemes.

\subsubsection{Diffusion Filtering}

Dirk-Jan used anisotropic diffusion filtering [42] as an iterative filtering approach for edge preserving smoothing in medical images, which can also be used to enhance the edges in a corneal image, reduce noise and preserve the nerve structure. The structure of the local image required in diffusion filtering is described using a structure tensor $\boldsymbol{J}(\boldsymbol{\nabla I})$, (also referred to as a "second-moment matrix") given by Eq.1. More details can be found in [43].

$$
J(\nabla I)=K_{j} *\left(\nabla I \cdot \nabla I^{T}\right)
$$

Here, $\boldsymbol{\nabla}$ is the del or nabla operator, $\boldsymbol{\nabla} \boldsymbol{I}$ is the image gradient and $\boldsymbol{K}_{\boldsymbol{j}}$ denotes a Gaussian kernel and $*$ is the convolution operator. The local orientation of the image is obtained from the eigen decomposition of the structure tensor:

$$
J(\nabla I)=\left[\begin{array}{ll}
V_{1} & V_{2}
\end{array}\right] \cdot\left[\begin{array}{cc}
\mu_{1} & 0 \\
0 & \mu_{2}
\end{array}\right] \cdot\left[\begin{array}{c}
V_{1}{ }^{T} \\
V_{2}{ }^{T}
\end{array}\right]
$$

where $\boldsymbol{V}_{1}, \boldsymbol{V}_{2}$ are eigenvectors that give the orientation of the local image with $\boldsymbol{V}_{\mathbf{1}}=\left[\begin{array}{ll}\boldsymbol{v}_{\mathbf{1 1}} & \boldsymbol{v}_{\mathbf{1 2}}\end{array}\right]^{T}$, etc., and the eigenvalues, with $\boldsymbol{\mu}_{1} \geq \boldsymbol{\mu}_{2}$ can be used to describe the average contrast in these directions. This structure tensor is used to define the diffusion tensor $\boldsymbol{D}$, which can be defined as follows [44]:

$$
\frac{\partial u}{\partial t}=\nabla \cdot(D \nabla u)
$$

where $\boldsymbol{u}(\boldsymbol{u}=\boldsymbol{u}(\boldsymbol{t}, \boldsymbol{x}, \boldsymbol{y}))$ is the image, $\boldsymbol{t}$ the diffusion time and $\boldsymbol{x}, \boldsymbol{y}$ are the pixel coordinates and $\boldsymbol{\nabla} \boldsymbol{u}$ is the gradient of the image $\boldsymbol{u}$. In general, the diffusion tensor $\boldsymbol{D}$ has the same eigenvectors set as given by the structure tensor:

$$
D=\left[\begin{array}{ll}
D_{11} & D_{12} \\
D_{12} & D_{22}
\end{array}\right] \text { with } D i j=\sum_{n=1.2} \lambda_{n} v_{n i} v_{n j}
$$

In Eq.2 and Eq.4, we notice the symmetry between $\lambda_{1}, \lambda_{2}$, which are the eigenvalues of the diffusion tensor and the structure tensor. Due to the nature of the corneal image, which consists of planar and tubular structures, the HDCS is used in this work. Depending on the local corneal image structure, the HDCS switches between CED and EED, the former if the local structure is tubular, or the latter if the local structure is planar.

\subsubsection{Hybrid Diffusion with Continuous Switch (HDCS)}

In general, medical images have complex structures with different intensities, shapes and sizes. If the EED is applied to a corneal confocal image, it filters the noise and enhances the edges and curves of the image, but blurs small structures. On the other hand, if the CED is applied, it enhances line-like structures such as nerves and preserves small structures [40]. Therefore, a new filter combining the advantages of both EED and CED could lead to better results. As mentioned before, a structure tensor is used to construct the diffusion tensors for the EED and CED. 


\begin{tabular}{|c|c|c|}
\hline$\frac{b_{i-1, j}-b_{i, j+1}}{4}$ & $\frac{c_{i, j+1}+c_{i, j}}{2}$ & $\frac{b_{i+1, j}+b_{i, j+1}}{4}$ \\
\hline$\frac{a_{i-1, j}+a_{i, j}}{2}$ & $-\frac{a_{i-1, j}+2 a_{i, j}+a_{i+1, j}}{2}+\frac{c_{i, j-1}+2 c_{i, j}+c_{i, j+1}}{2}$ & $\frac{a_{i+1, j}+a_{i, j}}{2}$ \\
\hline$b_{i-1, j}+b_{i, j-1}$ & $\frac{c_{i, j-1}+c_{i, j}}{2}$ & $\frac{b_{i+1, j}-b_{i, j-1}}{4}$ \\
\hline
\end{tabular}

\section{(a)}

\begin{tabular}{|c|c|c|}
\hline $\begin{aligned} & \frac{\left|b_{i-1, j+1}\right|-b_{i-1, j+1}}{4} \\
+ & \frac{\left|b_{i, j}\right|-b_{i, j}}{4}\end{aligned}$ & $\frac{c_{i, j+1}+c_{i, j}}{2}-\frac{\left|b_{i, j+1}\right|+\left|b_{i, j}\right|}{2}$ & $\begin{array}{l}\frac{\left|b_{i+1, j+1}\right|+b_{i+1, j+1}}{4} \\
+\frac{\left|b_{i, j}\right|+b_{i, j}}{4}\end{array}$ \\
\hline $\begin{array}{c}\frac{a_{i-1, j}+a_{i, j}}{2} \\
-\frac{\left|b_{i-1, j}\right|+\left|b_{i, j}\right|}{2}\end{array}$ & $\begin{array}{l}-\frac{a_{i-1, j}+2 a_{i, j}+a_{i+1, j}}{2} \\
-\frac{\left|b_{i-1, j+1}\right|-b_{i-1, j+1}+\left|b_{i+1, j+1}\right|+b_{i+1, j+1}}{4} \\
-\frac{\left|b_{i-1, j-1}\right|+b_{i-1, j-1}+\left|b_{i+1, j-1}\right|-b_{i+1, j-1}}{4} \\
+\frac{\left|b_{i-1, j}\right|+\left|b_{i+1, j}\right|+\left|b_{i, j-1}\right|+\left|b_{i, j-1}\right|+2\left|b_{i, j}\right|}{2} \\
-\quad \frac{c_{i, j-1}+2 c_{i, j}+c_{i, j+1}}{2}\end{array}$ & $\begin{array}{c}\frac{a_{i+1, j}+a_{i, j}}{2} \\
-\frac{\left|b_{i+1, j}\right|+\left|b_{i, j}\right|}{2}\end{array}$ \\
\hline $\begin{array}{l}\frac{b_{i-1, j-1}+b_{i-1, j-1}}{4} \\
+\frac{\left|b_{i, j}\right|+b_{i, j}}{4}\end{array}$ & $\frac{c_{i, j-1}+c_{i, j}}{2}-\frac{\left|b_{i, j-1}\right|+\left|b_{i, j}\right|}{2}$ & $\begin{array}{l}\frac{\left|b_{i+1, j-1}\right|-b_{i+1, j-1}}{4} \\
+\frac{\left|b_{i, j}\right|-b_{i, j}}{4}\end{array}$ \\
\hline
\end{tabular}

(b)

Figure 4. Notations for (a) the standard discretization scheme and (b) the non-negative discretization scheme.

Achilleas and Reiner [45] have proposed a discrete switch form to integrate EED and CED based on the difference $\left(\boldsymbol{\mu}_{1}-\boldsymbol{\mu}_{3}\right)$ of the structure tensor eigenvalues. However, the proposed filter cannot be applied properly in some situations. On the other hand, Adriënne and et al. [40] have proposed a Hybrid Diffusion with Continuous Switch (HDCS) by continuously combining the intermediate geometries of the EED and CED. The eigenvalues of the proposed hybrid diffusion tensor $\left(\boldsymbol{\lambda}_{\boldsymbol{h}_{\boldsymbol{l}}}\right)$ are adjusted to be a linear combination of the eigenvalues of the EED $\left(\boldsymbol{\lambda}_{\boldsymbol{c}_{\boldsymbol{t}}}\right)$ and CED $\left(\boldsymbol{\lambda}_{c_{l}}\right)$, which are given as follows:

$$
\left(\lambda_{h_{i}}\right)=(1-\varepsilon) \cdot\left(\lambda_{c_{i}}\right)+\varepsilon \cdot\left(\lambda_{c_{i}}\right)
$$

where $(\varepsilon)$ refers to the EED fraction which switches between using the eigenvalues of the EED eigenvalue $(\boldsymbol{\varepsilon} \rightarrow \mathbf{1})$ or the CED eigenvalue $(\boldsymbol{\varepsilon} \rightarrow \mathbf{0})$ diffusion tensor. In this paper, Hybrid Diffusion with Continuous Switch (HDCS) is proposed to enhance the structure of the corneal sub-basal nerves and reduce the unwanted noise. Therefore, the CED should be applied first, to preserve small structures and followed by EED, to reduce the noise isotropically. Details on HDCS can be found in [40].

\subsubsection{Diffusion Schemes}

The diffusion tensor equation (Eq.3) can be solved numerically using finite differences methods, using central differences instead of the spatial differences, and a forward difference approximation to discretize $\partial \boldsymbol{u} / \partial \boldsymbol{t}$ [45], as shown in Eq.6. The result is the basic structure of an explicit scheme, which can be used to compute the values at a new time level from the previous level as shown in Eq.7:

$$
\begin{gathered}
\frac{u_{i, j}^{k+1}-u_{i, j}^{k}}{\mathcal{T}}=A_{i, j}^{k} * u_{i, j}^{k} \\
u_{i, j}^{k+1}=\left(I+\mathcal{T} A_{i, j}^{k}\right) * u_{i, j}^{k}
\end{gathered}
$$

Here, $\boldsymbol{T}$ refers to the time step size and $\boldsymbol{u}_{\boldsymbol{i}, \boldsymbol{j}}^{\boldsymbol{k}}$ refers to the approximation of $\boldsymbol{u}(\boldsymbol{x}, \boldsymbol{t})$ in pixel $(\boldsymbol{i}, \boldsymbol{j})$ at time $\boldsymbol{k}_{\mathcal{T}}$. The notation $\boldsymbol{A}_{i, j}^{\boldsymbol{k}} * \boldsymbol{u}_{i, j}^{\boldsymbol{k}}$ is a discretization of the diffusion tensor expression. In this paper, three schemes have been investigated. These are the standard discretization scheme [46], the nonnegative discretization scheme [47] and the optimized scheme [42]. Fig.4 (a) and (b) show the stencil representations of the standard discretization and the non-negative discretization for $\boldsymbol{A}_{i, j}^{\boldsymbol{k}}$, assuming that the pixels have length $\mathbf{1}$ in both directions. Here $\boldsymbol{a}, \boldsymbol{b}$ and $\boldsymbol{c}$ are the output of the diffusion tensor $\boldsymbol{D}$. The last scheme is the optimized scheme for rotational invariant structures proposed by Dirk-Jan et al, for which more details to optimize the image derivatives in a numerical way can be found in [42]. In this paper, the coherence filter has been applied over four iterations, three in the preprocessing stage and one at the beginning of the edge detection stage. Each has been executed using a different diffusion scheme trying to enhance the structure of the corneal nerve without losing important information. In the preprocessing stage, the parameters have been set empirically, as follows: Diffusion Time $=2$, Diffusion Time Step size $=0.5, \boldsymbol{\sigma}=$ $8, \mathrm{D}=$ HDCS, with the diffusion scheme set to standard discretization in the first iteration and set to nonnegative discretization in the second and third iterations. In fact, all the parameter values in this step were chosen after an intensive empirical study investigating their influence on corneal images of different degrees of resolution. For example, we set the temporal step to be equal to 2, given that for lower values than 2, the produced image still has some noise, which results in detecting unwanted segments (e.g. small cells). Whereas for higher values than 2, a highly smoothed image will be produced with a large number of discontinues nerves. An example of a filtered image is shown in Fig.5 (b). The output of the coherence filter is smoothed further using a 2D-Gaussian filter, to reduce false artefacts, obtain a better corneal image for the edge detection stage and enhance the corneal image quality, with the result 
shown in Fig.5(c). The Gaussian filter [48] modifies the input signal through convolution with a 2D-Gaussian function defined as follows:

$$
G(x, y)=\frac{1}{2 \pi \sigma^{2}} e^{-\frac{x^{2}+y^{2}}{2 \sigma^{2}}}
$$

where $\boldsymbol{x}$ and $\boldsymbol{y}$ are the distances from the origin along the horizontal and vertical axes respectively, and $\sigma$ (set to 2) is the standard deviation of the Gaussian distribution.

\subsection{Morphological Operations Stage}

The main purpose of this stage is to describe the nerve structure more accurately by removing imperfections (e.g. various types of noise that can affect the nerve's structure) and make the nerve more visible. In this stage, the opening and erosion operations are used. Morphological operations are also used in other places in the proposed system to eliminate unwanted areas (small segments) without affecting the overall shape of the nerve. Generally, the morphological operations require two inputs, the input image that is to be processed and a structuring element (for more details see [49]). The dilation and erosion operations are the basic operations used in most morphological operations and are defined as follows:

$$
\begin{gathered}
f \oplus B=\max _{u, v}\left(f_{(x-u, y-v)}+B_{(u, v)}\right) \\
f \Theta B=\min _{u, v}\left(f_{(x+u, y+v)}-B_{(u, v)}\right)
\end{gathered}
$$

where $\boldsymbol{f}$ is a greyscale image $\boldsymbol{B}$ is a structuring element. $\boldsymbol{f} \oplus \boldsymbol{B}$ and $\boldsymbol{f} \boldsymbol{\Theta} \boldsymbol{B}$ represent a dilation and erosion, respectively. The opening and closing operations are defined in terms of the dilation and erosion operations, in Eq.11 and Eq.12, respectively:

$$
\begin{aligned}
& f o B=(f \Theta B) \oplus B \\
& f \cdot B=(f \oplus B) \Theta B
\end{aligned}
$$

In this stage, the values of the parameters used in this stage were selected empirically after a number of experiments were carried out using corneal sub-basal images with different levels of noise and illumination, taken into account to enhance the structure of the corneal nerve without losing important information in the whole image rather than a specific region of interest (ROI). The morphological opening operation is carried out on the image output from the Gaussian filter, using a disk shaped structure element of $\mathbf{4}$ pixels radius, followed by a background subtraction operation to separate out foreground objects from the background and detect the corneal nerves correctly. Then, an image contrast enhancement procedure is applied to enhance nerves visibility and to enhance the illumination uniformity of the corneal image by stretching the overall contrast of the image between two predefined lower and upper cutoffs which are empirically set to be 0.55 , and 0.999 , respectively. Finally, a morphological erosion operation using a structure element of $\mathbf{1}$ pixel is applied to refine the shapes of the corneal nerves, as shown in Fig.5 (d). Objects of $\mathbf{1}$ pixel size are discarded.

\subsection{Edge Detection Stage}

The edge detection process is used here to preserve useful structural information about nerve boundaries, and to drastically reduce unwanted areas. The main implemented approaches in the edge detection stage are shown in Fig.6. Firstly, the coherence filter is applied again for further enhancement and removal of noise introduced by the morphological stage, which can affect the accuracy of the nerve detection in the subsequent stages. In this step, the parameters of the coherence filter have been set empirically as follows: Diffusion Time $=1$, Diffusion Time Step size $=0.1, \boldsymbol{\sigma}=8, \mathrm{D}=\mathrm{HDCS}$, and the diffusion scheme $=$ Optimized Derivative Kernels Scheme. The corneal nerves are then detected by applying a Canny edge detector [50]. This first applies a Gaussian filter to the image to reduce noise and then calculates the gradient magnitude and direction at each pixel of the smoothed image. A non-maximum suppression algorithm is then applied using the gradient magnitude and direction and the local maxima of the gradient magnitude is detected as an edge pixel using a hysteresis threshold algorithm. The motivation behind the optimization of the Canny edge detector was to achieve the following desirable properties: minimizing the probability of multiple responses to a single edge; minimizing the probability of missed edge; minimizing the distance between the detected edge pixels and the actual edge. All these criteria play a significant role in addressing the issues of detecting and localizing the corneal nerves correctly. Further refinement is required to preserve the accurate thickness of the detected nerves, to remove some noisy background pixels, and to connect discontinuities in nerves in the segmented image. A morphological dilation operation is applied firstly using two line shaped structural elements, with lengths of $\mathbf{3}$ and angles of $\mathbf{9 0}^{\circ}$ and $\mathbf{0}^{\mathbf{}}$, respectively. Secondly, a morphological erosion operation is applied twice using structure element of one pixel size, to refine the shapes of the detected nerves. This is followed by a candidates' selection procedure based on the properties of the connected objects in the refined image where only objects that have a total area of more than $\mathbf{1 5 0}$ pixels are retained. See Fig.5 (e). Then a nerve connection procedure is applied to connect the discontinuous nerves. The gaps in nerve structures and branches appear in segmented nerves as a result of low visibility of parts of the nerves or noise introduced into the corneal images, for example. In the present study, a new technique has been proposed to link the discontinuous nerves correctly, which is summarized as follows:

1. Take the skeleton form of the final segmented image and determine the end points of each nerve segment in the segmented image.

2. Determine a possible maximum gap size between the endpoint of each disconnected nerve and neighbouring nerve. Then a binary circular region of radius $=$ (maximum gap size) $/ 2$ is placed at the endpoint of each nerve segment. If the endpoints of two nerve segments are close to each other, a straight line will be drawn connecting these two segments within the area covered by the circular structure elements, as shown in Fig. 7 (b).

3. Finally, by thinning the resultant image, the overlapped circular structure elements at the endpoints of each segment will leave behind a line of pixels linking the two endpoints of the nerve. While, the isolated endpoints are restored to their original structure, as shown in Fig.7 (c).

This step has a significant effect on calculating the tortuosity of nerves, because nerves' discontinuities will affect the measurements of nerve length, and hence the calculated tortuosity of the nerve. The values of the parameters used in the system were selected empirically after a number of experiments were carried out using corneal sub-basal images with different levels of noise and illumination. 


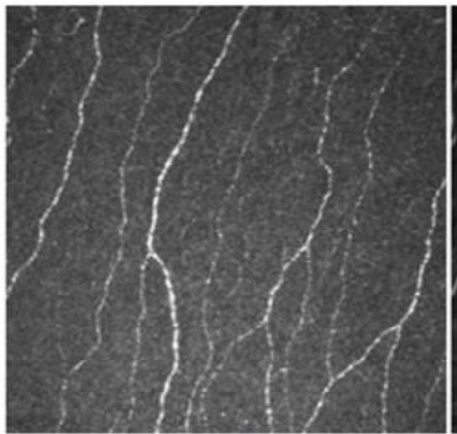

(a)

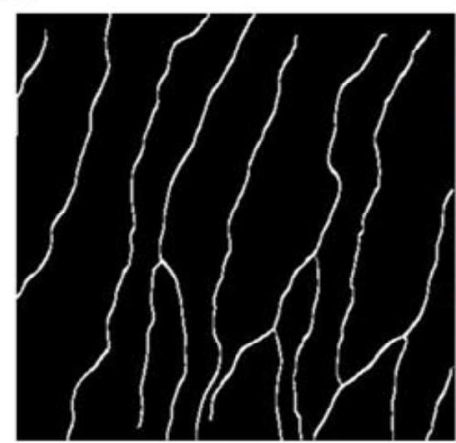

(e)

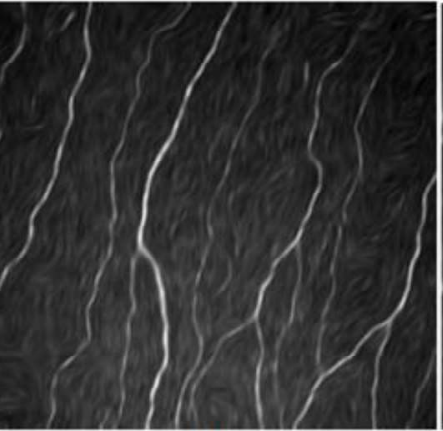

(b)

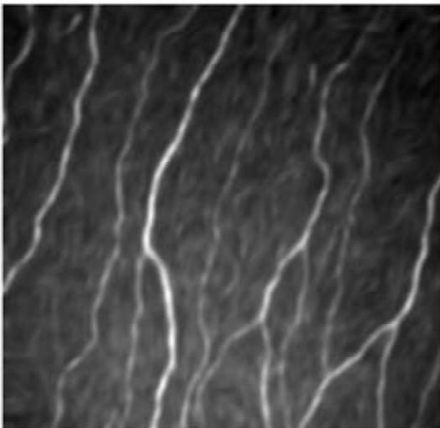

(c)

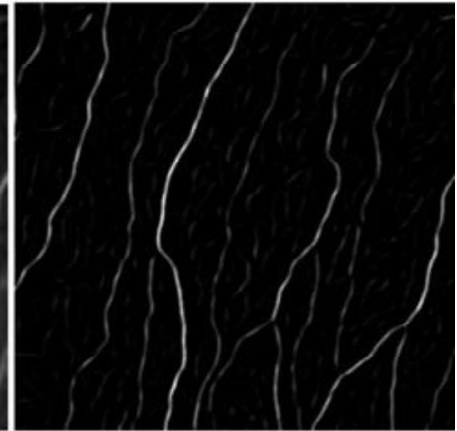

(d)

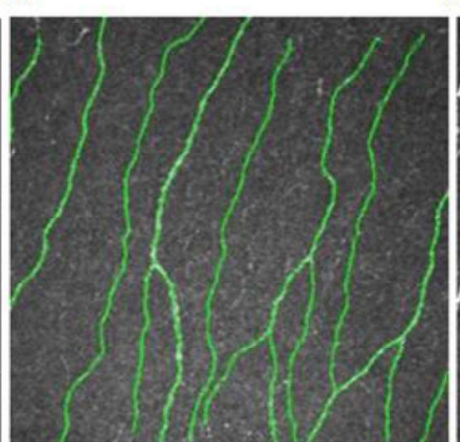

(f)

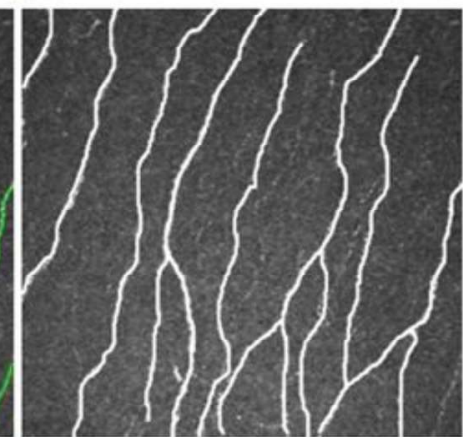

(g)

Figure 5. Corneal nerve segmentation system outputs: (a) Original corneal image, (b) Coherence filter output, (c) Gaussian filter output, (d) Morphological operations stage output, (e) Segmented image from the edge detection stage, (f) Automatically traced corneal sub-basal nerves, (g) Manually traced corneal sub-basal nerves.

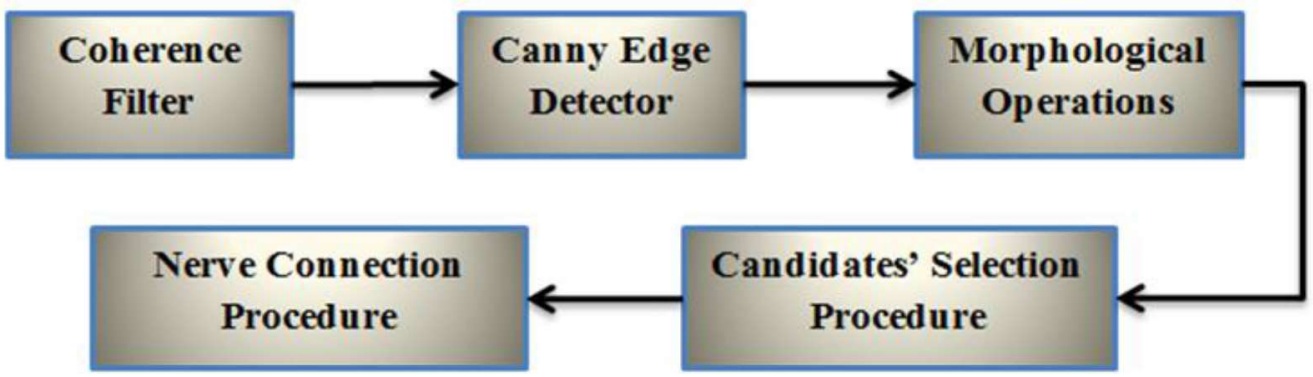

Figure 6. The main implemented approaches in the edge detection stage.

\subsection{Morphometric Parameters Quantification Stage}

The morphometric parameters quantification stage extracts a set of features from the automatically traced corneal sub-basal nerves in an easy and objective way. These clinical features are measured and extracted automatically so the proposed system can serve as a clinically helpful diagnostic tool for the early detection and follow up of DPN from CCM images. In this paper, the set of morphological extracted features related to the cornea's state of health which are investigated are nerve thickness, length, density and tortuosity. Additional features, such as nerve perimeter, area, and image intensity were also calculated for internal use.

\subsubsection{Nerve Length}

The nerve length in $(\mathrm{mm})$ is calculated for each nerve segment by taking the skeleton form of the nerve and then finding the branch points in order to break up the length of nerve segment $(\boldsymbol{S})$ into $(\boldsymbol{b})$ branches as follows:

$$
S=s_{1}+s_{2}+\cdots+s_{b}
$$

Finally, the nerve length is calculated by summing the distance between consecutive pixels in the nerve segment, as follows:

$$
\text { Nerve } e_{\text {length }}=\sum_{i=1}^{N-1} \sqrt{\left(x_{i-1}-x_{i}\right)^{2}+\left(y_{i-1}-y_{i}\right)^{2}}
$$

where $N$ in pixels is the number of constituent pixels which is obtained from the nerve skeleton segment and $\left(\boldsymbol{x}_{\boldsymbol{i}}, \boldsymbol{y}_{\boldsymbol{i}}\right)$ are the pixels coordinate in the nerve segment.

\subsubsection{Nerve Density}

The corneal nerve density in pixels $/ \mathrm{mm}^{2}$ is computed by dividing the sum of the nerve pixels by the image area as follows:

$$
\text { Nerve }_{\text {density }}=\frac{\sum \text { The nerve pixels }}{\text { ImageArea }\left(\mathrm{mm}^{2}\right)}
$$



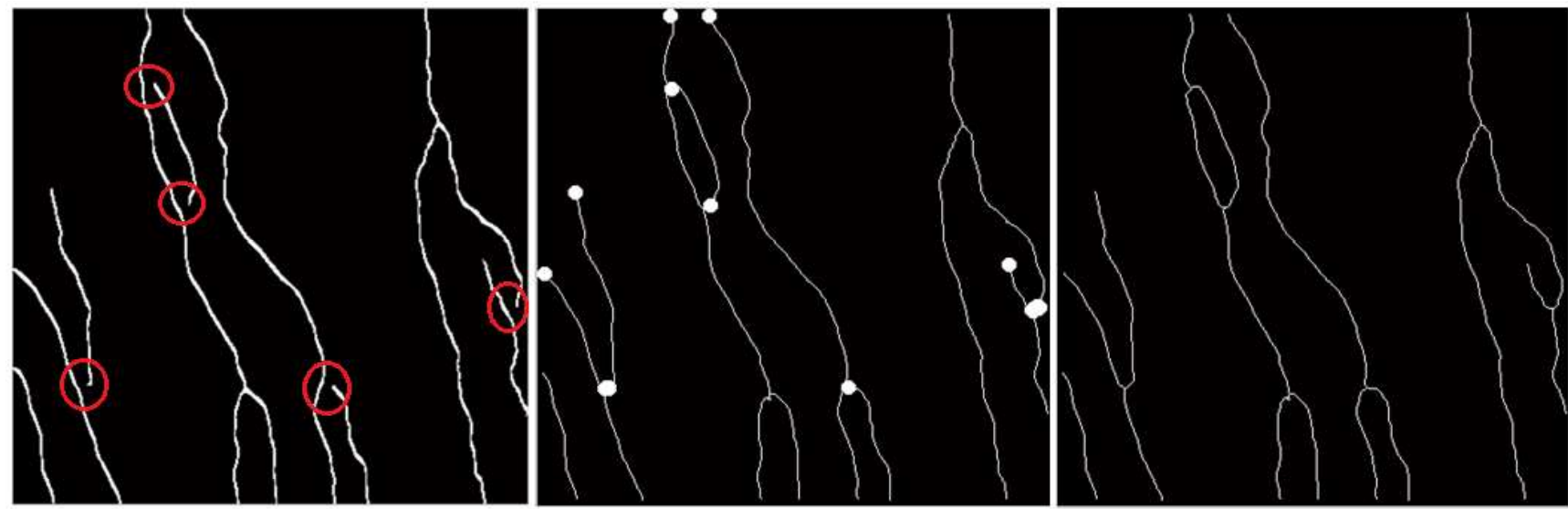

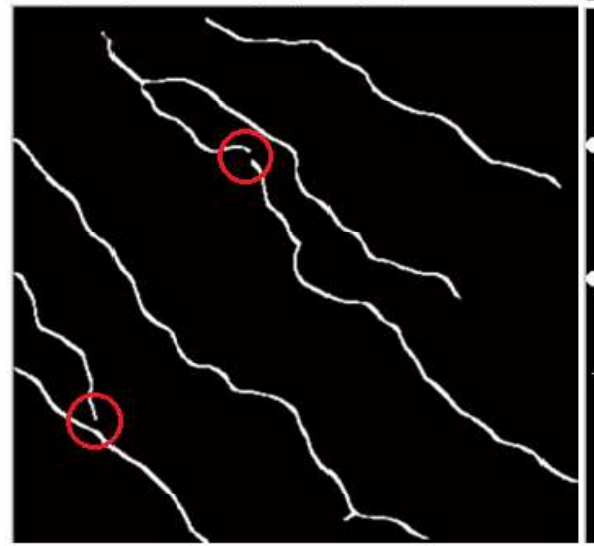

(a)

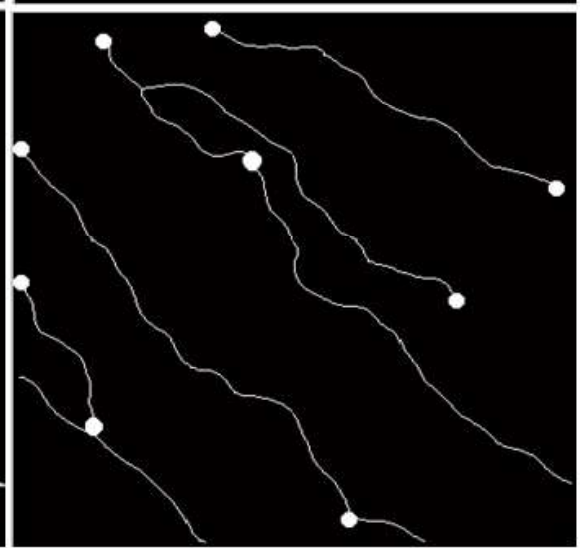

(b)

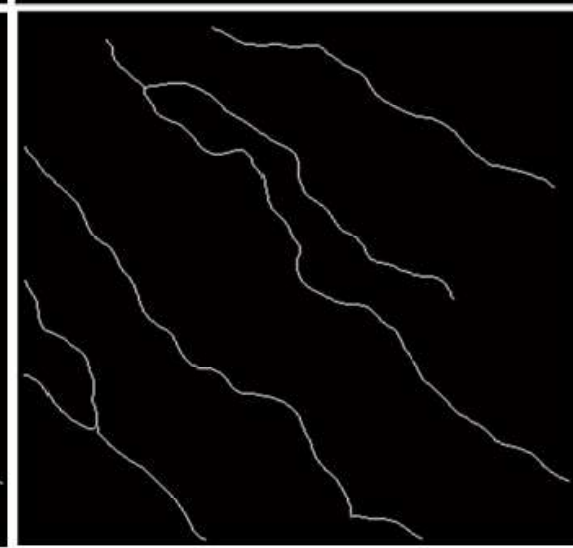

(c)

Figure 7. Nerves' connection procedure: (a) Two segmented images with disconnected nerves circled in red, (b) The binary circular structure element (white circles) drawn at the endpoints, and (c) Output image with connected nerves.

\subsubsection{Tortuosity Coefficient}

The Tortuosity Coefficient (TC) is used to gain information about the average curvature changes of the nerves. In this work, we calculate the average TC of the whole image and for each corneal nerve as well. Firstly, we calculate the length of each nerve segment $(\boldsymbol{S})$, as in (Section 3.5.1). Then, the TC index for nerve segment $(\boldsymbol{S})$ is then calculated as follows:

$$
\operatorname{TC}(S)=\sum_{n=1}^{b} s_{\text {length }}(n) / s_{\text {straight }}(n)
$$

where $\boldsymbol{s}_{\text {length }}$ is the branch length and is calculated by Eq.15. $\boldsymbol{s}_{\text {straight }}$ refers to the straight distance between the endpoints and is calculated as follows:

$$
s_{\text {straight }}=\sqrt{\left(x_{N}-x_{1}\right)^{2}+\left(y_{N}-y_{1}\right)^{2}}
$$

where $N$ is the number of constituent pixels obtained from the nerve skeleton branch and $(x, y)$ are pixels coordinate in the nerve branch. Finally, the average tortuosity of the whole image is obtained by computing the average tortuosity scores derived from each nerve.

\subsubsection{Nerve Thickness}

The nerve thickness in $(\mu \mathrm{m})$ is a measure of the average thickness of each corneal sub-basal nerve plexus, as shown in Fig.8. In this paper, a new algorithm for calculating the nerve thickness is proposed. The main steps of the proposed algorithm after labeling each nerve are as follows:
1. The distance transform is applied to the binary segmented image to calculate the Euclidean distance between each pixel in a nerve to the closest background pixel. In other words, for each nerve pixel, the distance from that particular pixel to the closest boundary pixel of the nerve is calculated.

2. Regarding distance values produced by the distance transform as heights in a $2 \mathrm{D}$ surface, the highest nerve pixels will be located along a ridge in the middle of the nerve segment. The distance values, associated with the half-way line in between the nerve segment are collected with some tolerance due to floating point arithmetic.

3. Finally, the average of all collected distances determines the half-width of the nerve segment. Hence, the full thickness can be calculated by multiplying the result obtained by 2 .

\section{Experimental Results}

The performance of the corneal sub-basal nerve segmentation system was evaluated initially on the first database containing 498 images where 238 images were taken from $\mathbf{8}$ healthy control subjects and the rest were taken from 12 diabetic patients with associated ground-truth as indicated previously in (Section 3.1). The evaluation is based on the calculation of the four quantitative performance measures: Structural SIMilarity Index (SSIM) [51], Probabilistic Rand Index (PRI) [52], Variation of Information (VoI) [53], and Global Consistency Error (GCE) [54].These metrics are widely used in the literature for evaluating the performance of segmentation systems and are defined as follows: 


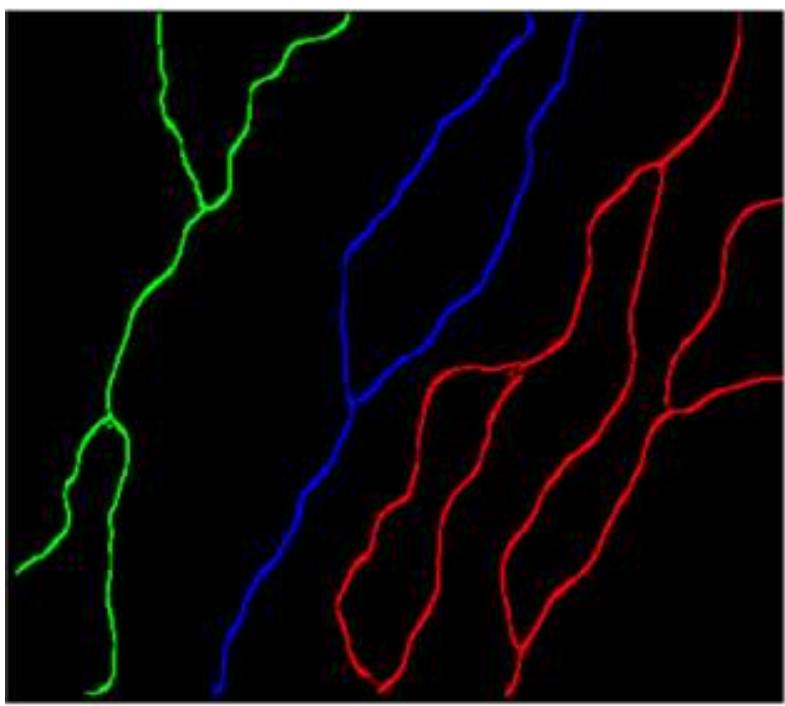

(a)

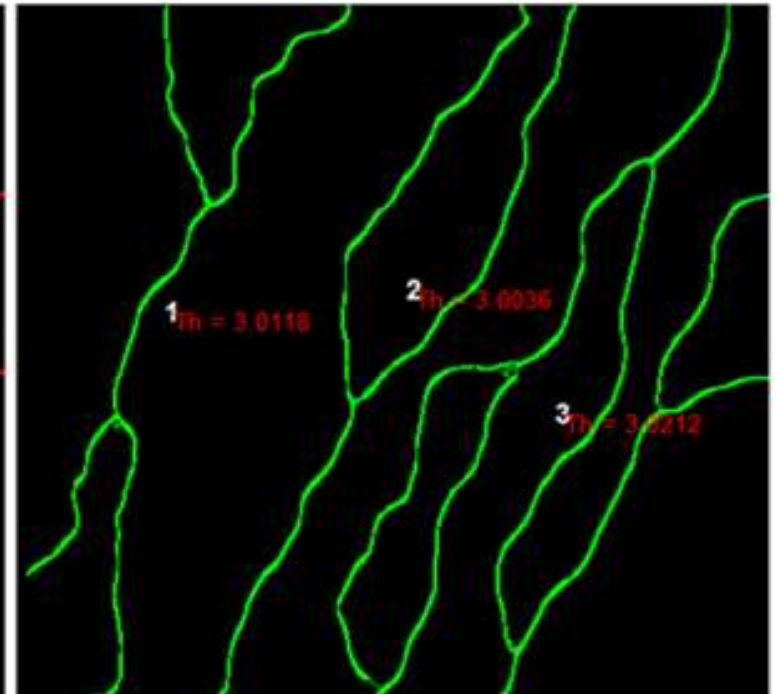

(b)

Figure 8. The thickness algorithm output: (a) Labeling of corneal nerves 1, 2 and 3 in green, blue and red, respectively, (b) Image map for the corneal nerves with their average thickness values indicated.

1. The Structural SIMilarity Index is an image quality assessment algorithm which measures the structural similarity index between the segmented image and a ground-truth image. The measurement compares three components: luminance, contrast and structure between the segmented image $(\mathbf{X})$ and the ground-truth image $(\mathbf{Y})$ within local window as follows:

$$
\operatorname{SSIM}(x, y)=\frac{\left(2_{\mu_{x} \mu_{y}}+C_{1}\right)\left(2_{\sigma_{x y}}+C_{2}\right)}{\left(\mu_{x}^{2}+\mu_{y}^{2}+C_{1}\right)\left(\sigma_{x}^{2}+\sigma_{y}^{2}+C_{2}\right)}
$$

Here, $\left(\boldsymbol{\mu}_{\boldsymbol{x}}\right.$ and $\left.\boldsymbol{\mu}_{\boldsymbol{y}}\right)$ and $\left(\boldsymbol{\sigma}_{x}^{2}\right.$ and $\left.\boldsymbol{\sigma}_{\boldsymbol{y}}^{\mathbf{2}}\right)$ are the mean intensities and the standard deviations of $\boldsymbol{x}$ and $\boldsymbol{y}$, respectively. $\sigma_{x y}$ is a covariance measure for $\boldsymbol{x}$ and $\boldsymbol{y} . \boldsymbol{C}_{1}=\left(\boldsymbol{k}_{1} \boldsymbol{L}\right)^{2}, \boldsymbol{C}_{2}=\left(\boldsymbol{k}_{2} \boldsymbol{L}\right)^{2}$ are small constants used to maintain stability when either $\left(\boldsymbol{\mu}_{\boldsymbol{x}}^{2}+\boldsymbol{\mu}_{y}^{2}\right)$ or $\left(\sigma_{x}^{2}+\sigma_{y}^{2}\right)$ is very close to zero; $\boldsymbol{L}$ represents the dynamic range of the pixel values (255 for 8-bit grayscale images) and $\boldsymbol{k}_{1}, \boldsymbol{k}_{2}<\boldsymbol{1}$. In this paper, $\boldsymbol{k}_{1}$ and $\boldsymbol{k}_{2}$ are set to the default values 0.04 and $\boldsymbol{L}$ is set at 100. The local measurements of $\boldsymbol{\mu}_{x}, \boldsymbol{\mu}_{\boldsymbol{y}}$ and $\boldsymbol{\sigma}_{\boldsymbol{x} y}$ are found within a local $(8 \times 8)$ square window, which moves pixel by pixel over the whole image and at each step the local measurements and SSIM are computed within the local window. In this work, the overall quality measure of the entire image is obtained by calculating the mean of SSIM as follows:

$$
\operatorname{MSSIM}(X, Y)=\frac{1}{M} \sum_{i=1}^{M} \operatorname{SSIM}\left(x_{i}, y_{i}\right)
$$

where $\boldsymbol{x}_{\boldsymbol{i}}$ and $\boldsymbol{y}_{\boldsymbol{i}}$ are the image contents at the $\boldsymbol{i}$-th local window, $\boldsymbol{M}$ is the number of local windows in the image and the MSSIM value ranges between $\mathbf{0}$ and $\mathbf{1}$; a higher value indicates greater similarity. Moreover, the SSIM index map can be obtained to provide a measurement of the local image quality over space, where a brighter SSIM index map indicates a better quality of segmentation, as shown in Fig.9 (c).
2. The Probabilistic Rand Index calculates the number of the fraction of pairs of pixels between the segmented and the ground-truth images whose labels are harmonious, through averaging across a set of ground truth images to account for scale variation in human perception. The PRI value ranges between $\mathbf{0}$ and $\mathbf{1}$, and a higher value indicates greater similarity.

3. The Variation of Information metric is a nonnegative metric that measures the distance between automatic and manual segmentations in terms of the information difference between them. The VoI metric depends on entropy and mutual information to calculate the distance between two clustering. The VoI between segmented image $(\boldsymbol{S})$ and the ground-truth image $\left(\boldsymbol{S}^{\prime}\right)$ is given by Eq.20, where a lower VoI value points to greater similarity.

$$
\operatorname{VoI}\left(S, S^{\prime}\right)=H(S)+H\left(S^{\prime}\right)-2 I\left(S, S^{\prime}\right)
$$

Here $\boldsymbol{V o I}$ ranges between $\mathbf{0}$ and $\infty, \boldsymbol{H}$ and $\boldsymbol{I}$ represent the entropy and the mutual information, respectively. In this work, the mutual information of $(\boldsymbol{S})$ and $\left(\boldsymbol{S}^{\prime}\right)$ can be calculated as follows:

$$
I\left(S, S^{\prime}\right)=\sum_{k=1}^{k} \sum_{k^{\prime}=1}^{k^{\prime}} P\left(k, k^{\prime}\right) \log \frac{P\left(k, k^{\prime}\right)}{P(k) P\left(k^{\prime}\right)}
$$

where $\boldsymbol{P}\left(\boldsymbol{k}, \boldsymbol{k}^{\prime}\right)$ is the joint probability distribution function of $(\boldsymbol{S})$ and $\left(\boldsymbol{S}^{\prime}\right)$, and $\boldsymbol{P}(\boldsymbol{k})$ and $\boldsymbol{P}\left(\boldsymbol{k}^{\prime}\right)$ are the marginal probability distribution functions of $(\boldsymbol{S})$ and $\left(\boldsymbol{S}^{\prime}\right)$, respectively.

4. The Global Consistency Error measures the extent to which the segmented image can be viewed as a refinement of the ground-truth image. Segmentations are considered to be consistent, if the segment is a set of pixels and a pixel is in an area of refinement, if the segment $(\boldsymbol{S})$ is a valid subset of segment $\left(\boldsymbol{S}^{\prime}\right)$. In this case, the local error is equal to zero; otherwise, if there is no relationship between the two segments, the two segments overlap in an inconsistent manner. The local refinement error between two segments is calculated as follows: 


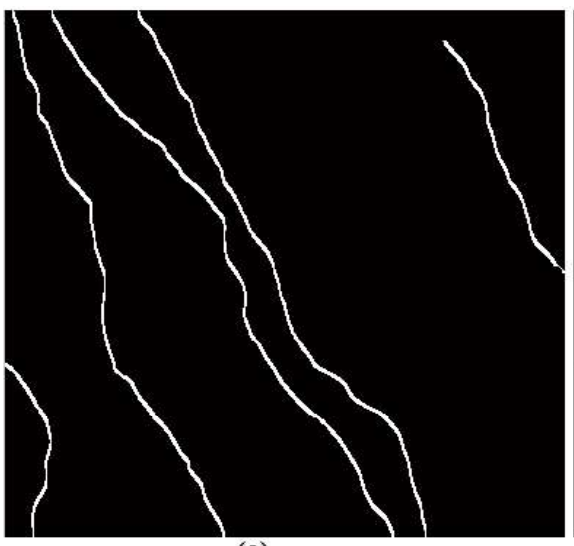

(a)

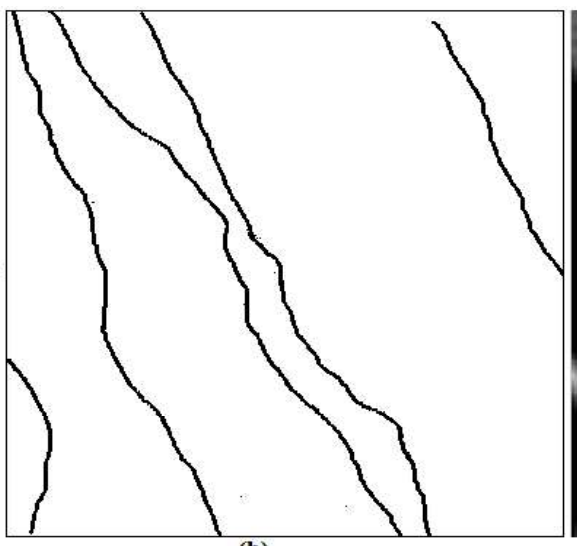

(b)

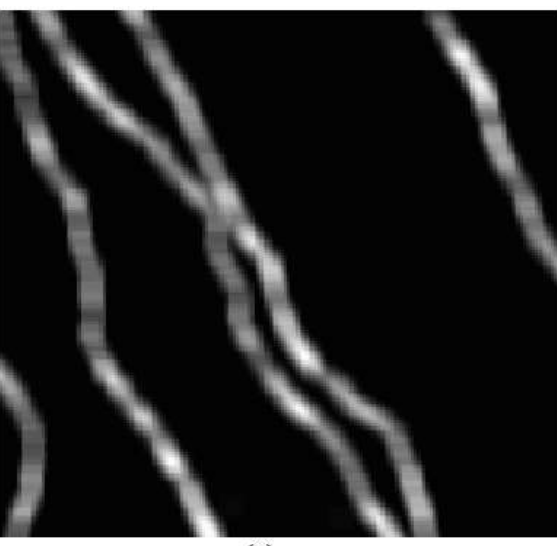

(c)

Figure 9. (a) A segmented image, (b) The binary form of ground-truth image, (c) The SSIM index map.

$$
E\left(S_{1}, S_{2}, p_{i}\right)=\frac{\left|R\left(S_{1}, p_{i}\right) \backslash R\left(S_{2}, p_{i}\right)\right|}{\left|R\left(S_{1}, p_{i}\right)\right|}
$$

where $\backslash$ denotes set difference, $\boldsymbol{S}_{1}$ and $\boldsymbol{S}_{2}$ are two segments. For a given pixel $\left(\boldsymbol{p}_{\boldsymbol{i}}\right)$, consider the segments that contain $\boldsymbol{p}_{\boldsymbol{i}}$ in $\boldsymbol{S}_{1}$ and $\boldsymbol{S}_{2}$. These sets of pixels are represented by $\boldsymbol{R}\left(\boldsymbol{S}_{1}, \boldsymbol{p}_{\boldsymbol{i}}\right)$ and $\boldsymbol{R}\left(\boldsymbol{S}_{2}, \boldsymbol{p}_{i}\right)$, respectively. The value of $\boldsymbol{E}\left(\boldsymbol{S}_{1}, \boldsymbol{S}_{2}\right.$, $\boldsymbol{p}_{\boldsymbol{i}}$ ) is zero when $\boldsymbol{S}_{\mathbf{1}}$ is a refinement of $\boldsymbol{S}_{2}$, but not vice versa. The GCE between segmented image $(\boldsymbol{S})$ and the ground-truth image $\left(\boldsymbol{S}^{\prime}\right)$ is given by Eq.23, and ranges between $\mathbf{0}$ and $\mathbf{1}$, a lower value being better.

$$
\operatorname{GCE}\left(S, S^{\prime}\right)=\frac{1}{n} \min \left\{\sum_{i} E\left(S, S^{\prime}, p_{i}\right), \sum_{i} E\left(S^{\prime}, S, p_{i}\right)\right\}
$$

The results obtained from the control subjects and the patient subjects are shown in Fig.10 and Fig.11, respectively. In these two figures, the overall average of each one of the four quantitative metrics is calculated for each subject in the dataset. The results obtained have demonstrated the robustness and effectiveness of the proposed nerve segmentation system, and the potentiality of using it as a fully automatic nerve tracing system to measure the morphological parameters for clinical diagnostic purposes, as a result of the high similarity rate obtained between the segmented images and the reference images.
In addition, the results obtained have demonstrated the ability of the proposed system to detect and trace the corneal nerves, effectively in real-time, with an execution time of about 7 seconds per image using a PC with Windows 8.1 operating system, a $1.80 \mathrm{GHz}$ Core i5-3337U CPU and 6 GB of RAM. The system code was written in MATLAB R2010a. At each stage of the proposed system, parameter values were selected after intensive experiments on a number of corneal sub-basal images with various degrees of degradation and illumination.

The overall performance of the proposed system was compared theoretically with other established automatic nerve detection systems $[55,29]$, due to the unavailability of the datasets used in these works. These systems are mainly based on using the single-scale and multi-scale dual-models to detect the nerve fibers. As shown in Fig.12, the output of the nerve detection system in [55] can result into a number of discontinuous nerve fibers that can significantly affect the calculation of the clinical features and this issue was tackled efficiently by the proposed system here as described in (Section 3.4). While, efficient performance has been demonstrated in [29] a training phase is required to train the adopted classifiers, which are used to classify the pixel to the fiber or non-fiber classes. However, promising results are provided by the proposed system for real-time requirements without any need for a training phase.

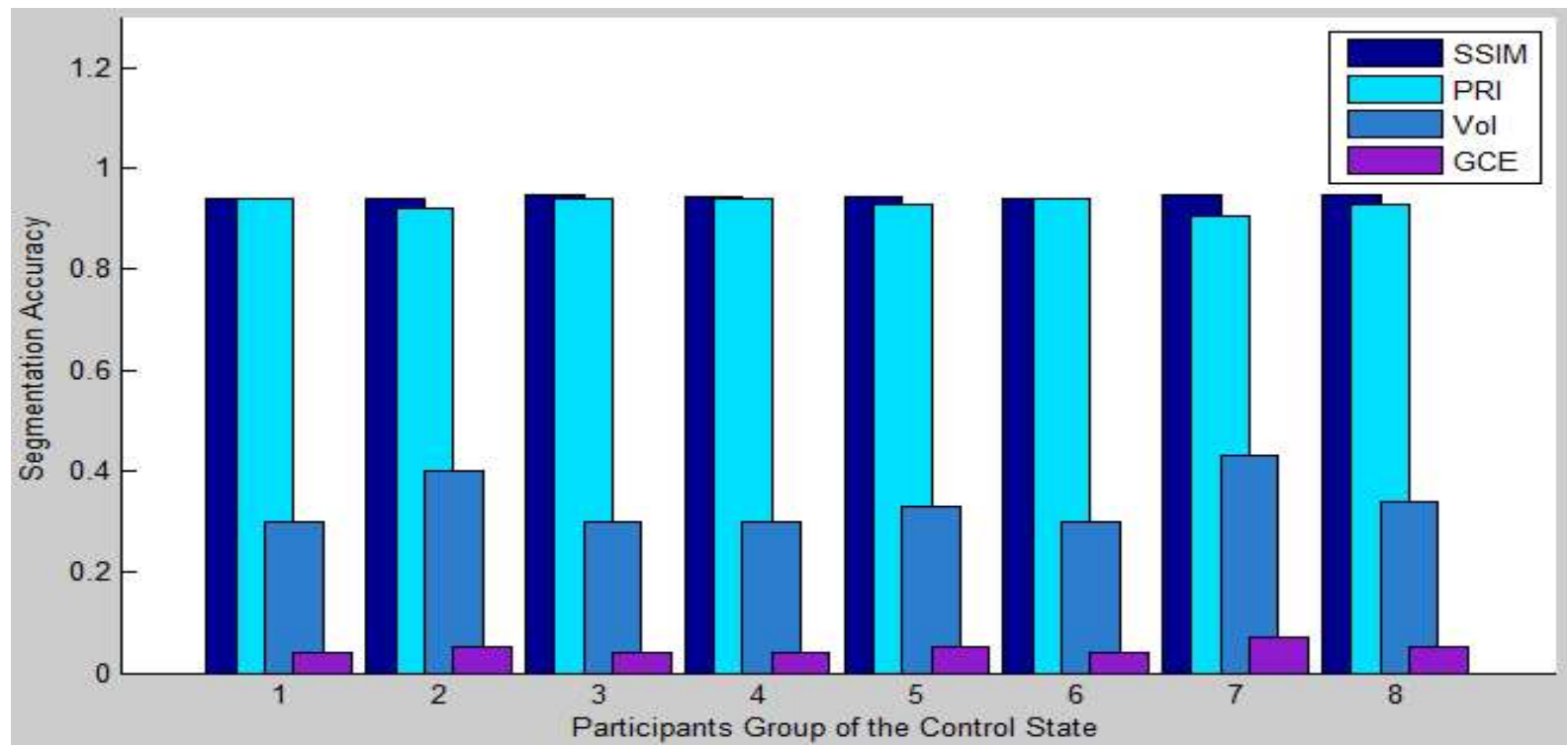

Figure 10. Descriptive statistics of the segmentation system performance of the Control group of the first database, where a higher value of SSIM and PRI is better and a lower value of VoI and GCE is better. 


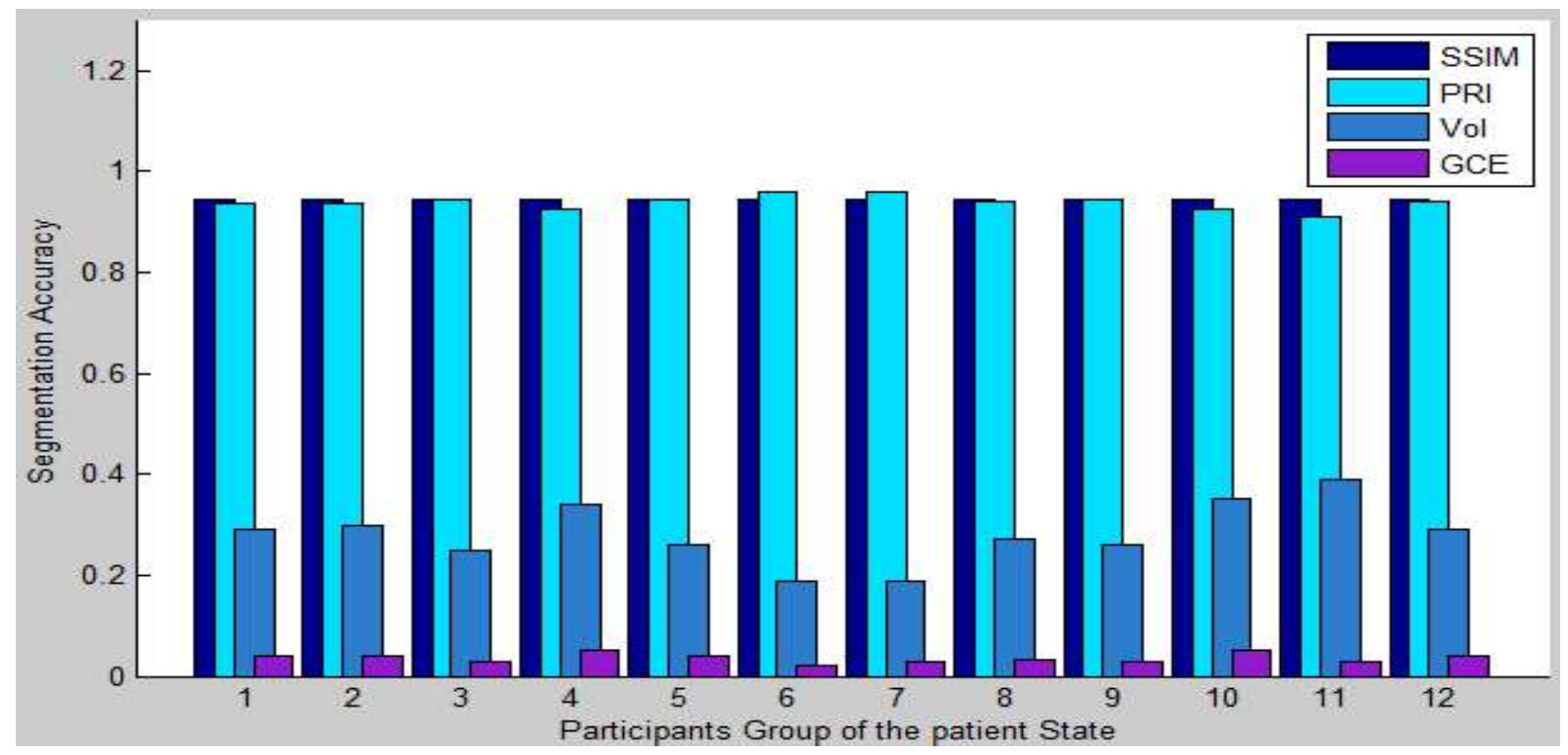

Figure 11. Descriptive statistics of the segmentation system performance of the patient group of the first database, where a higher value of SSIM and PRI is better and a lower value of VoI and GCE is better.
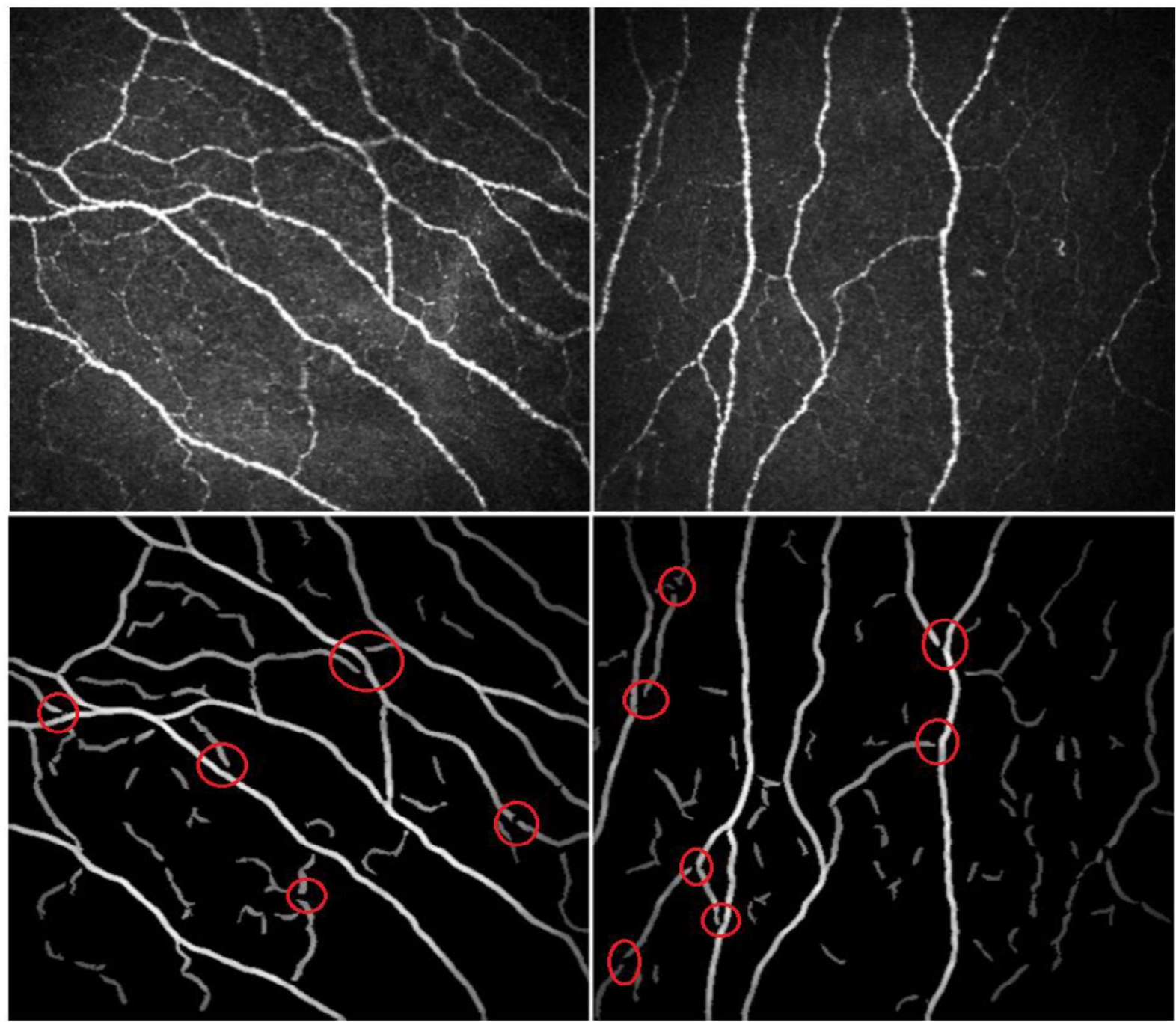

Figure 12. The output of the single scale dual-model detector: The images in the top row are the original images, while the bottom row is their response [29]. 
As mentioned before, the dataset was divided into four groups according to the severity of DPN. Therefore, clinical features, such as the average nerve tortuosity, the standard deviation of nerve tortuosity, the average nerve thickness, length and density are computed for each subject in the database after deriving the average for the whole image. This is followed by computing the overall average for each group, as summarized in Table (1). The extracted clinical features, obtained with the proposed system, are shown in Fig. 13.

There is a systematic decrease in nerve length and density and increase in nerve thickness and tortuosity associated with an increase in the severity of DPN, as shown in Table (1). However, the overall average nerve tortuosity did not give a useful estimation of the image tortuosity. This is because some images were classified by the ophthalmic clinicians as highly tortuous, because they contained just one or two nerves with several branches. Therefore, the average image tortuosity is low for these images, even though some images for each subject had relatively larger average values.
By applying an empirical threshold to these averages, the image that provides an average tortuosity higher than the predefined threshold is counted. The average of the counted image is then combined with the overall average tortuosity for each subject by applying this procedure, we found that the overall average tortuosity provides meaningful information relating to the DPN severity, as shown in Fig.14 (a) and solves the problem mentioned above. The most important clinical features are presented graphically in Fig.14. In this paper, the clinical features obtained by the proposed automatic system, except for nerve thickness were also compared against the ground truth manually traced by an experienced ophthalmologist. The main goal of this evaluation was to demonstrate the usefulness of the computed clinical features in differentiating control subjects from patients with diabetes and further differentiating diabetic patients in relations to the severity of neuropathy. The automated analysis of the proposed system presents equivalent results to the manual analysis, but the former is clearly quicker, more reliable and therefore clinically applicable. In this study, the execution time of the prototype was $\mathbf{1 3}$ seconds, starting from inputting the image until all the clinical features of each corneal nerve are obtained.

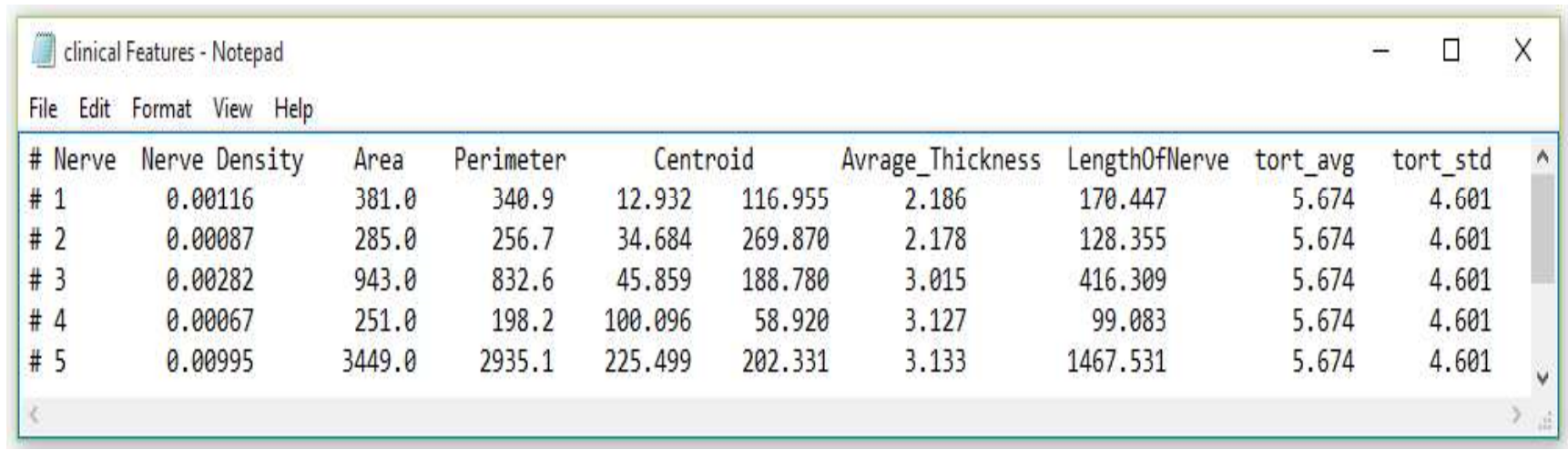

Figure 13. A readable text file format showing clinical features extracted from the first database.

Table 1. Summary of the descriptive clinical features of automatically traced nerves extracted from the first database.

\begin{tabular}{|c|c|c|c|c|c|c|c|c|c|}
\hline \multirow{3}{*}{ Groups } & \multicolumn{9}{|c|}{ Morphometric Parameters } \\
\hline & \multicolumn{4}{|c|}{ Manually Traced Nerves } & \multicolumn{5}{|c|}{ Automatically Traced Nerves } \\
\hline & $\begin{array}{l}\text { Ave. } \\
\text { Tort. }\end{array}$ & $\begin{array}{l}\text { STD } \\
\text { Tort. }\end{array}$ & $\begin{array}{l}\text { Ave. Length } \\
\text { (mm) }\end{array}$ & $\begin{array}{l}\text { Ave. Density } \\
(\text { Pixel/mm²) }\end{array}$ & $\begin{array}{l}\text { Ave. } \\
\text { Tort. }\end{array}$ & $\begin{array}{l}\text { STD } \\
\text { Tort. }\end{array}$ & $\begin{array}{l}\text { Ave. Length } \\
\text { (mm) }\end{array}$ & $\begin{array}{l}\text { Ave. Density } \\
\text { (Pixel/mm²) }\end{array}$ & $\begin{array}{l}\text { Ave. Thick. } \\
\qquad(\mu \mathrm{m})\end{array}$ \\
\hline Control & 8.27 & 7.18 & 60.92 & 0.0262 & 6.70 & 5.60 & 61.22 & 0.0316 & 2.83 \\
\hline Absent & 20.11 & 19.04 & 60.58 & 0.0222 & 13.9 & 12.79 & 60.34 & 0.0243 & 2.83 \\
\hline Mild & 37.52 & 36.41 & 58.49 & 0.0188 & 29.32 & 28.36 & 56.87 & 0.0182 & 2.85 \\
\hline Moderate & 40.45 & 39.30 & 57.08 & 0.0158 & 51.76 & 50.64 & 56.63 & 0.0164 & 2.88 \\
\hline
\end{tabular}




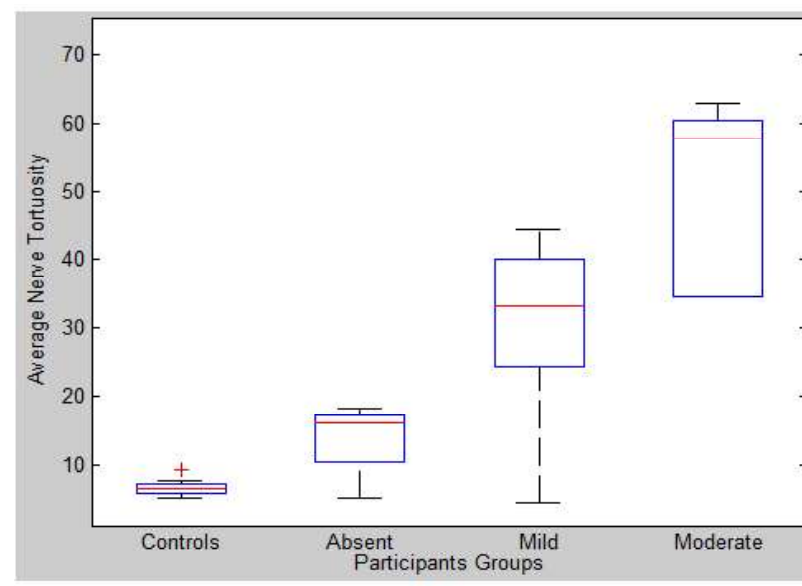

(a)

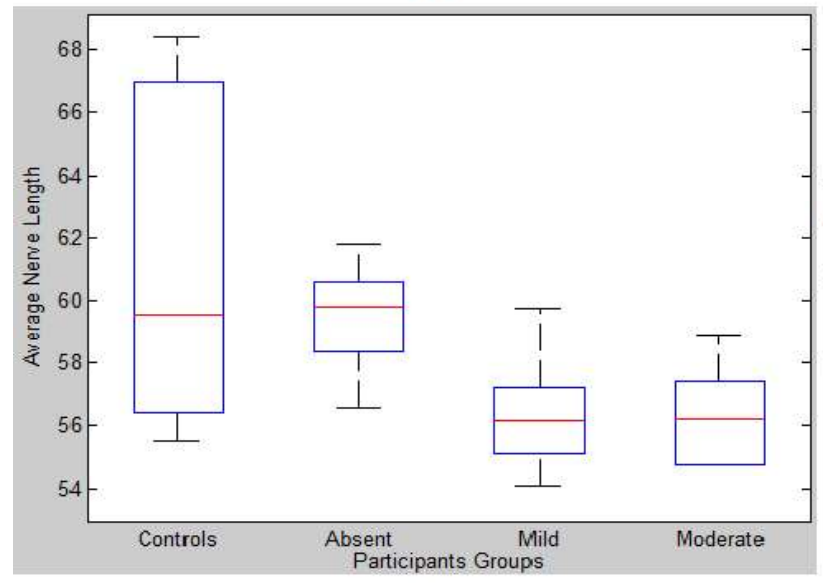

(c)

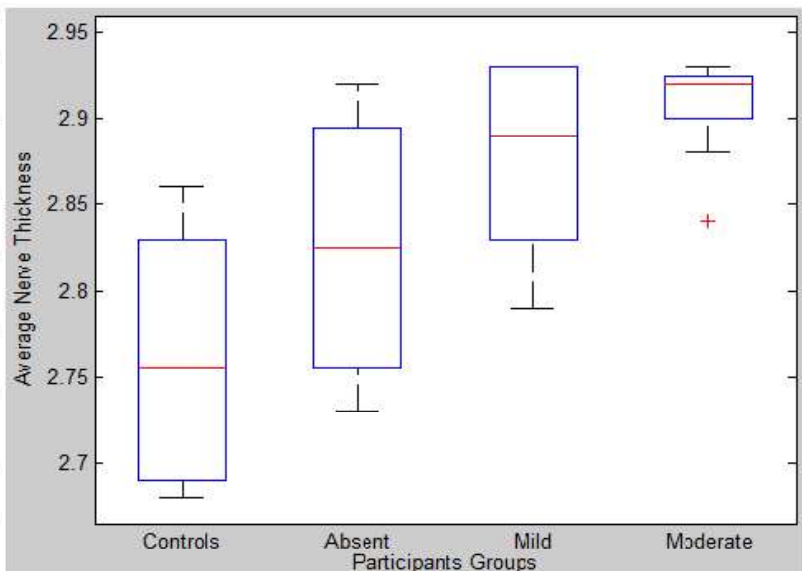

(b)

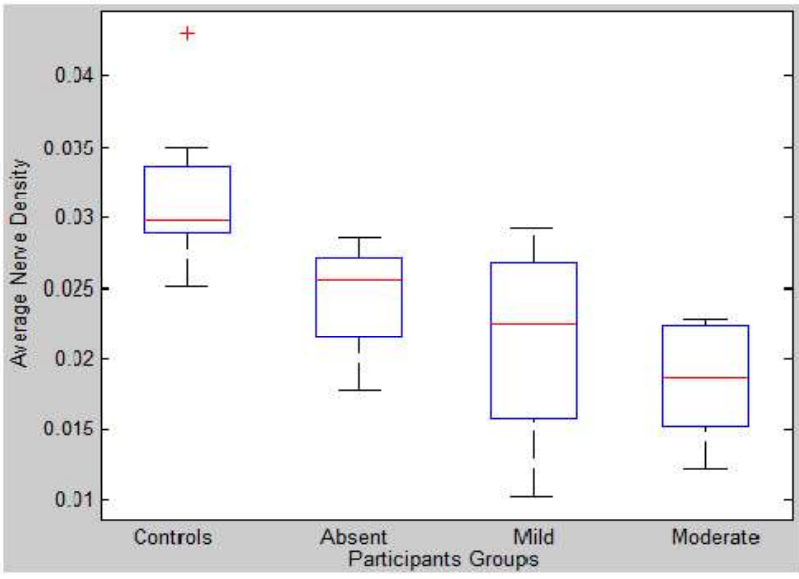

(d)

Figure 14. Representative box-plots with (median, inter-quartile range, outliers, and extreme cases of each parameter) illustrating the extracted clinical features from the first database: (a) Average Nerve Tortuosity, (b) Average Nerve thickness, and (c) Average Nerve Length, (d) Average Nerve Density.

To further evaluate and test the reliability and efficiency of the proposed system in extracting useful clinical features (e.g. nerve tortuosity, nerve thickness, nerve length and nerve density) and their relationship to DPN in a database of a total of 919 images taken from 172 individuals. The individuals were classified into controls, no neuropathy and neuropathy (See Section 3.1). The extracted clinical features using the proposed automated system are shown in Fig. 15. There was an increase in the average nerve tortuosity and thickness and decreases in the average nerve length and density with increasing severity of DPN.

\section{Conclusions and Future Work}

In this paper, a fully automatic, efficient real-time corneal sub-basal nerve segmentation and morphological parameter quantification system is proposed. Anisotropic diffusion and Gaussian filters were used to enhance the visibility of the nerve and to reduce the noise in the corneal image that can be caused by the acquisition process. In addition, an efficient technique is proposed to connect the discontinuous nerves. The results obtained have demonstrated the reliability and efficiency of the proposed segmentation system and the potential to use it as a real-time and a fully automatic nerve tracing system in patients with DPN as an early diagnostic and for follow-up. In the second stage, a number of useful clinical features, such as nerve length, density, thickness, and tortuosity as well as nerve perimeter, area and the image intensity were calculated. In this part, a new algorithm has been proposed to calculate the average nerve thickness. Our results have demonstrated the effects of DPN on the corneal sub-basal nerves, in terms of increased average nerve tortuosity and thickness coupled with decreased average nerve length and density. For example, from Table (1) one can see that the average nerve tortuosity and thickness have increased from 6.70 and 2.83 respectively, for the control group to 51.76 and 2.88 in the moderate group. On the other hand, the average nerve length and density have decreased from 60.92 and 0.0262 respectively for the control group to 57.08 and 0.0158 for the moderate group.

The results derived in this study are dependent on the successful connection of endpoints with neighbouring endpoints or branching nerves. As a future enhancement we would like to deploy larger circular regions to search for neighbouring endpoints/nerve regions and use decision making support (e.g. minimum Euclidean Distance) to tackle situations where more than one endpoint are being detected inside the circular region. In the present study the extracted clinical parameters were compared individually in all subjects to establish the efficiency of the imaging modules developed in this paper. However, successful diagnosis requires building general profiles for every subject by combining their available clinical information. Hence, a future research direction should include developing an automated machine learning-based system for diagnosing and differentiating control subjects from diabetic patients with and without neuropathy. To do so, we will need to build a learning module from the databases used in this research and feed them with the 4 additional parameters extracted from every subject (average nerve tortuosity, average nerve thickness, average nerve length, and average nerve density) to create a more comprehensive morphological phenotype in every subject, which we anticipate could lead to a more reliable diagnosis and stratification of severity of DPN. 


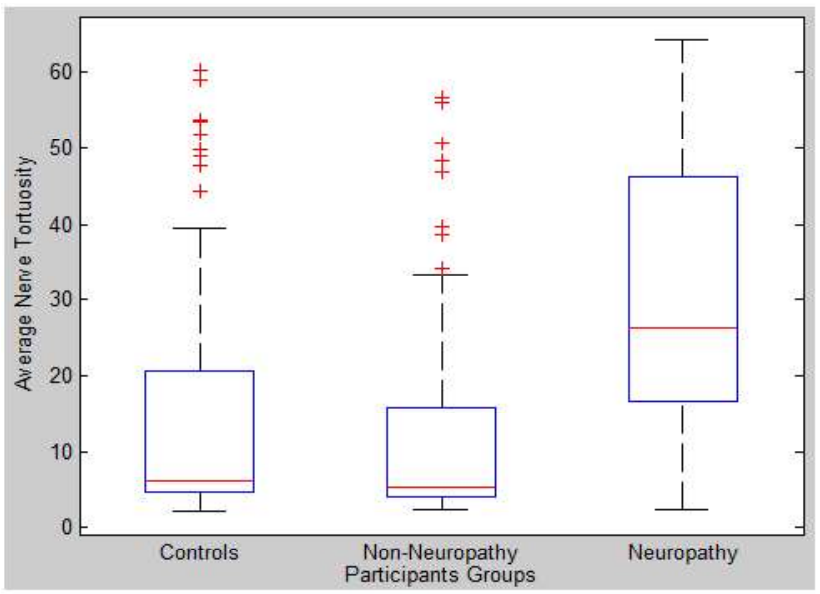

(a)

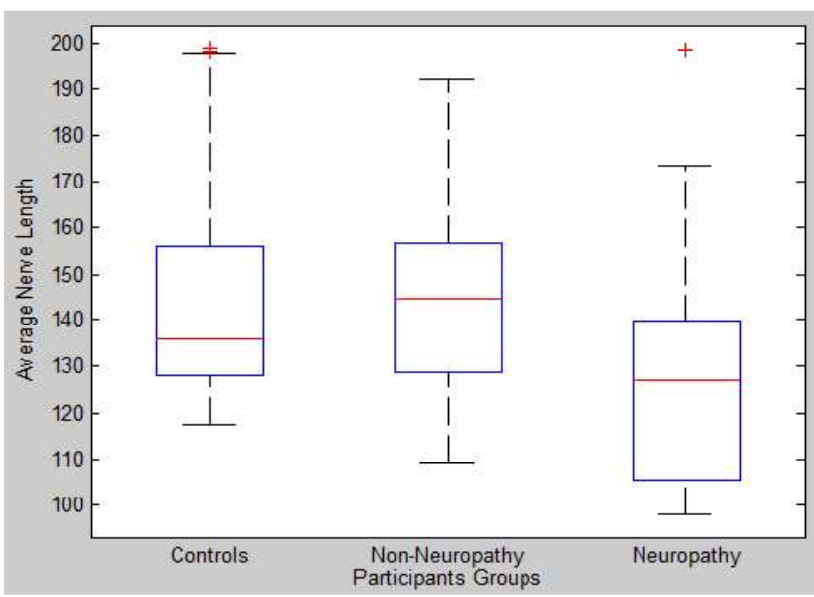

(c)

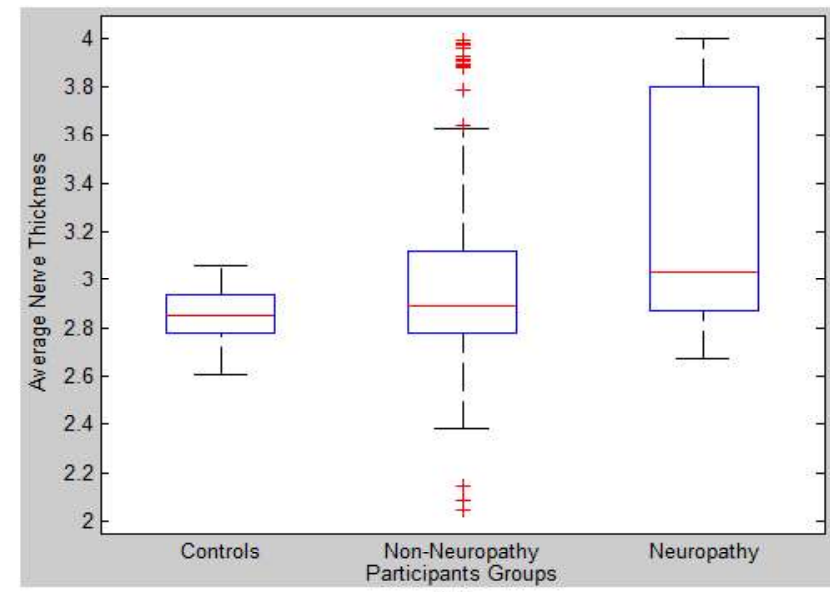

(b)

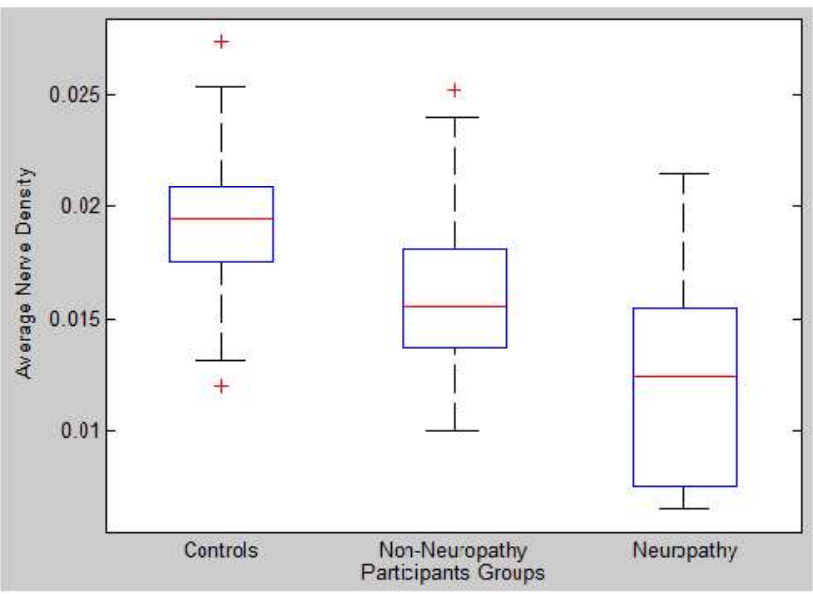

(d)

Figure 15. Representative box-plots with (median, inter-quartile range, outliers, and extreme cases of each parameter) illustrating the extracted clinical features from the second database: (a) Average Nerve Tortuosity, (b) Average Nerve Thickness (c) Average Nerve Length, and (d) Average Nerve Density.

\section{Acknowledgments}

The authors thank Dr. A. M. Morgado (University of Coimbra, Portugal) for kindly providing the CCM images with the manually traced ground-truth images for the first dataset. In addition, the authors thank Dr. Mhd Saeed Sharif for his effort in improving the first draft of this article.

\section{References}

[1] S. V. Patel, J. W. McLaren, D. O. Hodge, and W. M. Bourne, "Confocal microscopy in vivo in corneas of long-term contact lens wearers," Investig. Ophthalmol. Vis. Sci., vol. 43, no. 4, pp. 995-1003, 2002.

[2] Jalbert I, Stapleton F, Papas E, et al. "In vivo confocal microscopy of the human cornea," Br J Ophthalmol, vol. 87, pp. 225-236, 2003.

[3] G. Bitirgen, A. Ozkagnici, R. A. Malik, and R. Oltulu, "Evaluation of contact lens-induced changes in keratoconic corneas using in vivo confocal microscopy," Investig. Ophthalmol. Vis. Sci., vol. 54, no. January 2016, pp. 5385-5391, 2013.

[4] G. Bitirgen, A. Ozkagnici, B. Bozkurt, and R. A. Malik, "In vivo corneal confocal microscopic analysis in patients with keratoconus," Int J Ophthalmol, vol. 8, no. 3, pp. 534-539, 2015.

[5] J. a O. Moilanen, M. H. Vesaluoma, L. J. Müller, and T. M. T. Tervo, "Long-term corneal morphology after PRK by in vivo confocal microscopy," Investig. Ophthalmol. Vis. Sci., vol. 44, no. 3, pp. 10641069, 2003.

[6] K. Kurbanyan, L. M. Hoesl, W. a Schrems, and P. Hamrah, "Corneal nerve alterations in acute Acanthamoeba and fungal keratitis: an in vivo confocal microscopy study.," Eye (Lond)., vol. 26, no. 1, pp. 12632, Jan. 2012.
[7] R. L. Niederer, D. Perumal, T. Sherwin, and C. N. J. McGhee, "Corneal innervation and cellular changes after corneal transplantation: an in vivo confocal microscopy study.," Invest. Ophthalmol. Vis. Sci., vol. 48, no. 2, pp. 621-6, Feb. 2007.

[8] D. V Patel and C. N. J. McGhee, "Mapping the corneal sub-basal nerve plexus in keratoconus by in vivo laser scanning confocal microscopy.," Invest. Ophthalmol. Vis. Sci., vol. 47, no. 4, pp. 1348-51, Apr. 2006.

[9] L. S. Mannion, C. Tromans, and C. O'Donnell, "An evaluation of corneal nerve morphology and function in moderate keratoconus," Contact Lens Anterior Eye, vol. 28, no. 4, pp. 185-192, 2005.

[10] U. Alam, O. Asghar, I. N. Petropoulos, M. Jeziorska, H. Fadavi, G. Ponirakis, A. Marshall, M. Tavakoli, A. J. M. Boulton, N. Efron, and R. A. Malik, "Small Fiber Neuropathy in Patients With Latent Autoimmune Diabetes in Adults: Table 1," Diabetes Care, vol. 38, no. 7, pp. e102-e103, 2015.

[11] O. Asghar, I. N. Petropoulos, U. Alam, W. Jones, M. Jeziorska, A. Marshall, G. Ponirakis, H. Fadavi, A. J. M. Boulton, M. Tavakoli, and R. A. Malik, "Corneal Confocal Microscopy Detects Neuropathy in Subjects With Impaired Glucose Tolerance: Figure 1," Diabetes Care, vol. 37, no. 9, pp. 2643-2646, 2014.

[12] S. Azmi, M. Ferdousi, I. N. Petropoulos, G. Ponirakis, U. Alam, H. Fadavi, O. Asghar, A. Marshall, A. J. Atkinson, W. Jones, and et al. "Corneal Confocal Microscopy Identifies Small-Fiber Neuropathy in Subjects With Impaired Glucose Tolerance Who Develop Type 2 Diabetes," Diabetes Care, vol. 38, no. 8, pp. 1502-1508, 2015.

[13] G. Bitirgen, A. Ozkagnici, R. A Malik, and H. Kerimoglu, "Corneal nerve fibre damage precedes diabetic retinopathy in patients with type 2 diabetes mellitus.," Diabet. Med., vol. 31, no. 4, pp. 431-8, 2014.

[14] X. Chen, J. Graham, M. A. Dabbah, I. N. Petropoulos, G. Ponirakis, O. Asghar, U. Alam, A. Marshall, H. Fadavi, M. Ferdousi, and others, "Small Nerve Fiber Quantification in the Diagnosis of Diabetic Sensorimotor Polyneuropathy: Comparing Corneal Confocal Microscopy With Intraepidermal Nerve Fiber Density," Diabetes Care, no. January, 2015. 
[15] C. Dehghani, N. Pritchard, K. Edwards, D. Vagenas, A. W. Russell, R. A. Malik, and N. Efron, "Natural History of Corneal Nerve Morphology in Mild Neuropathy Associated With Type 1 Diabetes: Development of a Potential Measure of Diabetic Peripheral Neuropathy," Invest. Ophthalmol. Vis. Sci., vol. 55, no. 12, pp. 79827990, 2014.

[16] L. E. Lovblom, E. M. Halpern, T. Wu, D. Kelly, A. Ahmed, G. Boulet, A. Orszag, E. Ng, M. Ngo, V. Bril, and B. A. Perkins, "In Vivo Corneal Confocal Microscopy and Prediction of Future-Incident Neuropathy in Type 1 Diabetes : Preliminary Longitudinal Analysis.," Can. J. diabetes, vol. 39, pp. 390-397, 2015.

[17] D. Pacaud, K. G. Romanchuk, M. Tavakoli, C. Gougeon, H. Virtanen, M. Ferdousi, A. Nettel-Aguirre, J. K. Mah, and R. A. Malik, "The Reliability and Reproducibility of Corneal Confocal Microscopy in Children," Investig. Opthalmology Vis. Sci., vol. 56, no. 9, p. 5636 , 2015.

[18] C. Quattrini, M. Tavakoli, M. Jeziorska, P. Kallinikos, S. Tesfaye, J. Finnigan, A. Marshall, A. J. M. Boulton, N. Efron, and R. A. Malik, "Surrogate Markers of Small Fiber Damage in Human Diabetic Neuropathy," Diabetes, vol. 56, no. 8, pp. 2148-2154, 2007.

[19] I. N. Petropoulos, U. Alam, H. Fadavi, O. Asghar, P. Green, G. Ponirakis, A. Marshall, A. J. M. Boulton, M. Tavakoli, and R. a. Malik, "Corneal nerve loss detected with corneal confocal microscopy is symmetrical and related to the severity of diabetic polyneuropathy," Diabetes Care, vol. 36, no. 11, pp. 3646-3651, 2013.

[20] S. Al-fahdawi, R. Qahwaji, A. S. Al-waisy, and S. Ipsopn, "An Automatic Corneal Subbasal Nerve Registration System Using FFT and Phase Correlation Techniques for an Accurate DPN diagnosis," IEEE International Conference on Computer and Information Technology; Ubiquitous Computing and Communications; Dependable, Autonomic and Secure Computing; Pervasive Intelligence and Computing, pp. 1035-1041, 26-28 Oct. 2015.

[21] A. Elbita, R. Qahwaji, S. Ipson, M. S. Sharif, and F. Ghanchi, "Preparation of 2D sequences of corneal images for 3D model building," Comput. Methods Programs Biomed., vol. 114, no. 2, pp. 194-205, 2014.

[22] M. Saeed, R. Qahwaji, S. Hayajneh, R. Alzubaidi, S. Ipson, and A Brahma, "An Efficient System For Preprocessing Confocal Corneal Images For Subsequent Analysis," Computational Intelligence (UKCI), 14th UK Workshop on, pp.1-8, 8-10 Sept. 2014.

[23] M. S. Sharif, R. Qahwaji, E. Shahamatnia, R. Alzubaidi, S. Ipson, and A. Brahma, "An efficient intelligent analysis system for confocal corneal endothelium images," Comput. Methods Programs Biomed., vol. 122, no. 3, pp. 421-436, 2015.

[24] N. Pritchard, K. Edwards, A. W. Russell, B. a Perkins, R. a Malik, and N. Efron, "Corneal confocal microscopy predicts 4-year incident peripheral neuropathy in type 1 diabetes.," Diabetes Care, vol. 38, no. 4, pp. 671-5, 2015

[25] M. Tavakoli, M. Mitu-Pretorian, I. N. Petropoulos, H. Fadavi, O. Asghar, U. Alam, G. Ponirakis, M. Jeziorska, A. Marshall, N. Efron, A. J. Boulton, T. Augustine, and R. A. Malik, "Corneal Confocal Microscopy Detects Early Nerve Regeneration in Diabetic Neuropathy After Simultaneous Pancreas and Kidney Transplantation," Diabetes, vol. 62 , no. 1 , pp. 254-260, 2013.

[26] I. Otel, P. Cardoso, L. Gomes, S. Gouveia, S. F. Silva, J. P. Domingues, J. S. Silva, M. Carvalheiro, M. J. Quadrado, and A. M. Morgado, "Diabetic Peripheral Neuropathy Assessment Through Corneal Nerve Morphometry," no. February, pp. 1-6, 2013.

[27] I. N. Petropoulos, T. Manzoor, P. Morgan, H. Fadavi, O. Asghar, U. Alam, G. Ponirakis, M. a Dabbah, X. Chen, J. Graham, M. Tavakoli, and R. a Malik, "Repeatability of in vivo corneal confocal microscopy to quantify corneal nerve morphology.," Cornea, vol. 32, no. 5, pp. e83-9, 2013

[28] A. Ferreira, A. M. Morgado, and J. S. Silva, "Corneal nerves segmentation and morphometric parameters quantification for early detection of diabetic neuropathy," IFMBE Proc., vol. 29, pp. 264-267, 2010.

[29] M. a Dabbah, J. Graham, I. N. Petropoulos, M. Tavakoli, and R. a Malik, "Automatic analysis of diabetic peripheral neuropathy using multi-scale quantitative morphology of nerve fibres in corneal confocal microscopy imaging.," Med. Image Anal., vol. 15, no. 5, pp. 738-47, Oct. 2011

[30] I. N. Petropoulos, U. Alam, H. Fadavi, A. Marshall, O. Asghar, M. A. Dabbah, X. Chen, J. Graham, G. Ponirakis, A. J. M. Boulton, M. Tavakoli, and R. A. Malik, "Rapid Automated Diagnosis of Diabetic Peripheral Neuropathy With In Vivo Corneal Confocal Microscopy," Investig. Opthalmology Vis. Sci., vol. 55, no. 4, p. 2071, 2014.

[31] Webolutions, (2015), Vision Care Specialists, Available: http://www.visioncarespecialists.com/services/medical- conditions/corneal-disease/, [Accessed:3 May 2015].

[32] A. Ruggeri, F. Scarpa, and E. Grisan, "Analysis of corneal images for the recognition of nerve structures," Annu. Int. Conf. IEEE Eng. Med. Biol. - Proc., pp. 4739-4742, 2006.

[33] E. Poletti and A. Ruggeri, "Automatic nerve tracking in confocal images of corneal subbasal epithelium," Proc. 26th IEEE Int. Symp. Comput. Med. Syst., pp. 119-124, Jun. 2013.

[34] A. Ferreira, A. M. Morgado, and J. S. Silva, "A method for corneal nerves automatic segmentation and morphometric analysis.," Comput. Methods Programs Biomed., vol. 107, no. 1, pp. 53-60, Jul. 2012.

[35] F. Scarpa, E. Grisan, and A. Ruggeri, "Automatic recognition of corneal nerve structures in images from confocal microscopy.," Invest. Ophthalmol. Vis. Sci., vol. 49, no. 11, pp. 4801-7, Nov. 2008.

[36] F. Scarpa, X. Zheng, Y. Ohashi, and A. Ruggeri, "Automatic evaluation of corneal nerve tortuosity in images from in vivo confocal microscopy.," Invest. Ophthalmol. Vis. Sci., vol. 52, no. 9, pp. 6404-8, Aug. 2011.

[37] M. A. Dabbah, J. Graham, M. Tavakoli, Y. Petropoulos, and R. A. Malik, "Nerve Fibre Extraction in Confocal Corneal Microscopy Images for Human Diabetic Neuropathy Detection using Gabor Filters," in Medical Image Understanding and Analysis (MIUA), 2009.

[38] P. Guimar, J. Wigdahl, E. Poletti, and A. Ruggeri, "A fully-automatic fast segmentation of the sub-basal layer nerves in corneal images *," pp. 5422-5425, 2014.

[39] R. Annunziata, A. Kheirkhah, S. Aggarwal, B. M. Cavalcanti, P. Hamrah, and E. Trucco, "Tortuosity Classification of Corneal Nerves Images Using a Multiple-Scale-Multiple-Window Approach," vol. 44, no. $0,2014$.

[40] A. M. Mendrik, E.-J. Vonken, A. Rutten, M. a Viergever, and B. van Ginneken, "Noise reduction in computed tomography scans using 3-d anisotropic hybrid diffusion with continuous switch.," IEEE Trans. Med. Imaging, vol. 28, no. 10, pp. 1585-94, Oct. 2009.

[41] J. Weickert and J. Weickert, Anisotropic Diffusion in Image Processing. 1998.

[42] D. Kroon, C. H. Slump, and T. J. J. Maal, "Optimized Anisotropic Rotational Invariant Diffusion Scheme on Cone-beam CT." Medical Image Computing and Computer-Assisted Intervention-MICCAI. Springer Berlin Heidelberg, pp. 221-228, 2010

[43] Y. You, W. Xu, A. Tannenbaum, and M. Kaveh, Behavioral Analvsis of AnisotroDic Diffusion in Image Processing, vol. 5, no. 11. 1996.

[44] J. Weickert and H. Scharr, "A Scheme for Coherence-Enhancing Diffusion Filtering with Optimized Rotation Invariance," J. Vis. Commun. Image Represent., vol. 13, no. 1-2, pp. 103-118, Mar. 2002.

[45] a S. Frangakis and R. Hegerl, "Noise reduction in electron tomographic reconstructions using nonlinear anisotropic diffusion.," $J$. Struct. Biol., vol. 135, no. 3, pp. 239-50, Sep. 2001

[46] L. Fritz, "Diffusion-based applications for interactive medical image segmentation," Proc. CESCG (Central Eur. Semin. Comput. Graph., 2006.

[47] J. Weickert, "Anisotropic diffusion in image processing," Image Rochester NY, vol. 256, no. 3, p. 170, 1998.

[48] R. a. Haddad and A. N. Akansu, "A class of fast Gaussian binomial filters for speech and image processing," IEEE Trans. Signal Process., vol. 39 , no. 3 , pp. $723-727,1991$

[49] I. De, B. Chanda, and B. Chattopadhyay, "Enhancing effective depthof-field by image fusion using mathematical morphology," Image Vis. Comput., vol. 24, no. 12, pp. 1278-1287, Dec. 2006

[50] J. Canny, "A Computational Approach to Edge Detection," IEEE Trans. Pattern Anal. Mach. Intell., vol. PAMI-8, no. 6, pp. 679-698, Nov. 1986.

[51] Z. Wang, A. C. Bovik, H. R. Sheikh, S. Member, E. P. Simoncelli, and S. Member, "Image Quality Assessment: From Error Visibility to Structural Similarity," vol. 13, no. 4, pp. 1-14, 2004.

[52] J. Kaur, S. Agrawal, and R. Vig, "Integration of Clustering, Optimization and Partial Differential Equation Method for Improved Image Segmentation," Int. J. Image, Graph. Signal Process., vol. 4, no. 11 , pp. 26-33, 2012.

[53] M. Meil, "Comparing clusterings — an information based distance," vol. 98, pp. 873-895, 2007

[54] D. Martin, C. Fowlkes, D. Tal, and J. Malik, "A database of human segmented natural images and its application toevaluating segmentation algorithms and measuring ecological statistics," Proc. Eighth IEEE Int. Conf. Comput. Vision. ICCV 2001, vol. 2, no. July, 2001 .

[55] M. A. Dabbah, J. Graham, Petropoulos, and A. I. M. Tavakoli, and R.A. Malik, "Dual-Model Automatic Detection of Nerve-Fibres in Corneal Confocal Microscopy Images," in Changes, vol. 29, no. 6, pp. 997-1003, 2010. 